This item was submitted to Loughborough's Research Repository by the author.

Items in Figshare are protected by copyright, with all rights reserved, unless otherwise indicated.

\title{
Processing symbolic and non-symbolic proportions: Domain-specific numerical and domain-general processes in intraparietal cortex
}

PLEASE CITE THE PUBLISHED VERSION

https://doi.org/10.1016/j.brainres.2019.02.029

PUBLISHER

Elsevier

VERSION

AM (Accepted Manuscript)

\section{PUBLISHER STATEMENT}

This paper was accepted for publication in the journal Brain Research and the definitive published version is available at https://doi.org/10.1016/j.brainres.2019.02.029

LICENCE

CC BY-NC-ND 4.0

\section{REPOSITORY RECORD}

Mock, J, S Huber, J Bloechle, Julia Bahnmuller, Korbinian Moeller, and E Klein. 2019. "Processing Symbolic and Non-symbolic Proportions: Domain-specific Numerical and Domain-general Processes in Intraparietal Cortex". Loughborough University. https://hdl.handle.net/2134/12504485.v1. 
Processing symbolic and non-symbolic proportions:

Julia Mock ${ }^{\mathrm{a}}$, Stefan Huber ${ }^{\mathrm{a}}$, Johannes Bloechle ${ }^{\mathrm{a}, \mathrm{b}, \mathrm{e}}$, Julia Bahnmueller ${ }^{\text {a,d }}$,

${ }^{a}$ Leibniz-Institut für Wissensmedien, Schleichstraße 6, 72076 Tuebingen, Germany

$9 \quad{ }^{\mathrm{b}}$ Hertie-Institute for Clinical Brain Research, Division of Neuropsychology, Otfried-Müller-Straße 27, 72076

10 Tuebingen, Germany

$11{ }^{\mathrm{c}}$ Department of Psychology, Eberhardt-Karls University Tuebingen, Schleichstraße 4, 72076 Tuebingen, Germany

$12{ }^{\mathrm{d}}$ LEAD Graduate School, University of Tuebingen, Geschwister-Scholl-Platz, 72074 Tuebingen, Germany

$13{ }^{\mathrm{e}}$ Department of Psychiatry and Psychotherapy, University Hospital Tuebingen, Germany

17 Correspondence:

18 Julia Mock

19 Leibniz-Institut für Wissensmedien

20 Schleichstrasse 6

2172076 Tuebingen, Germany

22 Email: j.mock@iwm-tuebingen.de

23 Tel.: +49 7071 979-266 


\section{Abstract}

Previous studies on the processing of fractions and proportions focused mainly on the

3 processing of their overall magnitude information in the intraparietal sulcus (IPS). However, the

4 IPS is also associated with domain-general cognitive functions beyond processing overall 5 magnitude, which may nevertheless be involved in operating on magnitude information of

6 proportions. To pursue this issue, the present study aimed at investigating whether there is a shared

7 neural correlate for proportion processing in the intraparietal cortex beyond overall magnitude

8 processing and how part-whole relations are processed on the neural level. Across four presentation

9 formats (i.e., fractions, decimals, dot patterns, and pie charts) we observed a shared neural substrate

10 in bilateral inferior parietal cortex, slightly anterior and inferior to IPS areas recently found for

11 overall magnitude proportion processing. Nevertheless, when evaluating the neural correlates of

12 part-whole processing (i.e., contrasting fractions, dot patterns, and pie charts vs. decimals), we

13 found wide-spread activation in fronto-parietal brain areas. These results indicate involvement of

14 domain-general cognitive processes in part-whole processing beyond processing the overall

15 magnitude of proportions. The dissociation between proportions involving part-whole relations and

16 decimals was further substantiated by a representational similarity analysis, which revealed

17 common neural processing for fractions, pie charts, and dot patterns, possibly representing their

18 bipartite part-whole structure. In contrast, decimals seemed to be processed differently on the

19 neural level, possibly reflecting missing processes of actual proportion calculation in decimals.

21 Word count: 227 words

22 Keywords: proportions, symbolic, non-symbolic, fMRI, part-whole processing 


\section{Introduction}

Recent research indicated that processing natural numbers and proportions is associated with activation in the intraparietal sulcus (IPS; Piazza, Pinel, Le Bihan, \& Dehaene, 2007; Sokolowski, Fias, Mousa, \& Ansari, 2017; see Nieder, 2005 for a review). Furthermore, we recently showed that processing overall magnitude of symbolic and non-symbolic proportions draws on similar neural correlates in the IPS (Mock et al., 2018). However, the IPS is not specifically dedicated to number processing. As part of the higher order (also referred to as tertiary) association cortex, the IPS hosts a variety of fine-grained sensory, motor, and cognitive functions including attention orienting, grasping, spatial working memory, saccade planning, mental rotation, and navigation (Culham and Kanwisher, 2001; Humphreys and Lambon Ralph, 2017, 2015; Simon et al., 2002).

However, joint aspects of processing proportions beyond overall magnitude have not yet been systematically investigated at the neural level. By the term beyond overall magnitude we refer to domain-specific numerical and domain-general processes involved in proportion processing as compared to the specific processing of overall proportion magnitude as reflected by the distance effect (Mock et al., 2018) and, thus, to processes not necessarily limited to magnitude processing. As such, the aim of the present study was to investigate shared aspects of processing proportions in the IPS reflecting domain-specific numerical as well as domain-general processes. In other words, we suggest that the neural activations evaluated in the present study do not necessarily reflect overall magnitude processing as indicated by the numerical distance effect (Mock et al., 2018). Nevertheless, processing magnitude information might still play an important role in proportion processing when operating on the actual proportion. This might, however, rather involve more top-down regulated proportion processing including domain-specific magnitude-related processes and other domain-general cognitive mechanisms. 
Processing proportions seems particularly suited for this purpose as their processing is more

2 difficult and requires more steps than just accessing number magnitude information. Furthermore,

3 we evaluated whether different presentation formats (i.e., symbolic and non-symbolic presentation

4 formats) of the same concept (i.e., proportions) share underlying domain-specific numerical as well

5 as domain-general neural processes.

6 In the following, we will first present recent neuroimaging evidence on the processing of

7 symbolic fractions and non-symbolic proportions. However, because neuroimaging studies in

8 numerical cognition have so far focused mainly on the IPS as the core area for number magnitude

9 processing, we then also introduce the role of the intraparietal cortex for more domain-general

10 cognitive processes that are not necessarily specific to processing proportion magnitude, such as

11 visuo-spatial processing, attention orienting, mental rotation, saccades, or spatial working memory.

\subsection{The neural underpinnings of (proportion) number processing}

14 Previous studies showed that a bilateral fronto-parietal network centered around the IPS is

15 associated with number processing (Piazza, Pinel, Le Bihan, \& Dehaene, 2007; see Nieder, 2005

16 for a review). Within this network, the IPS seems to be specifically involved in the processing of

17 numerical magnitude information (Piazza et al., 2007; Pinel et al., 2001) and mental calculation

18 (Arsalidou and Taylor, 2011; Dehaene et al., 1999). Furthermore, it was observed that bilateral

19 inferior parietal lobule (IPL) and precuneus, left superior parietal lobule (SPL) and right superior

20 frontal gyrus (SFG) are involved in processing of both symbolic and non-symbolic numbers

21 (Sokolowski et al., 2017). More specifically, left intraparietal cortex as well as the left angular

22 gyrus and right supramarginal gyrus seem to be involved in the processing of symbolic numbers,

23 exact calculation, and arithmetic fact retrieval (Dehaene et al., 1999; Grabner et al., 2009; Holloway

24 et al., 2010; Simon et al., 2002; Sokolowski et al., 2017; Venkatraman et al., 2005). In contrast, the 
1 right IPS seems to underlie a semantic representation of numbers and an abstract code for absolute

2 and relative magnitude (Cohen Kadosh et al., 2007; Mock et al., 2018; Mussolin et al., 2013).

3 Additionally, a right-lateralized fronto-parietal network including right SPL and IPL, right SFG

4 and right middle occipital gyrus seem to be specifically activated in the processing of non-symbolic

5 numerosities (Holloway et al., 2010; Sokolowski et al., 2017). This suggests that symbolic and

6 non-symbolic numbers are processed using both overlapping and distinct neural substrates

7 (Sokolowski et al., 2017).

$8 \quad$ Furthermore, recent studies also revealed that brain regions typically associated with natural

9 number processing are also involved in processing proportions (Ischebeck et al., 2010, 2009a,

10 Jacob and Nieder, 2009a, 2009b). For instance, Jacob and Nieder (2009a) observed that the

11 processing of symbolic fraction magnitude within the bilateral IPS seems to be independent of the

12 presentation format and suggested that the same populations of neurons code the same fraction

13 magnitude. Importantly, Jacob and Nieder (2009b) observed similar intraparietal effects for non-

14 symbolic proportions. This is substantiated by a recent study, in which we found that processing

15 symbolic (i.e., fractions and decimals) and non-symbolic proportions (i.e., dot patterns and pie

16 charts) share a neural correlate in occipito-parietal regions including the right IPS (Mock et al.,

17 2018). Interestingly, in our study (Mock et al., 2018), joint IPS activation was observed to be

18 specific for the processing of overall symbolic and non-symbolic proportion magnitude (see also

19 Ischebeck et al., 2009a; DeWolf et al., 2016).

20 Thus, on the neural level proportion magnitude seems to be represented by its value as a

21 whole (i.e., its relative magnitude; Ischebeck et al., 2009a; Jacob \& Nieder, 2009b). Based on this

22 notion, DeWolf and colleagues (2016) investigated whether different symbolic numbers (i.e.,

23 fractions, integers, decimals) map onto a joint abstract magnitude code or whether different

24 representations exist for specific number types (integers versus symbolic proportions) or number 
1 notations (fractions vs. base- $10^{1}$, e.g. $1 / 2$ vs 0.5 ). Interestingly, processing fractions, integers, and

2 decimals activated areas in the IPS. However, fractions yielded a distinct activation pattern from

3 decimals and integers within the IPS, as revealed by both univariate and multivariate analyses.

4 Thus, neural processing seemed to be sensitive to number notation (fractions vs. base-10), but not

5 to number type (integer vs. proportion).

6 Taken together, previous work indicated that similar intraparietal brain regions are involved

7 in number magnitude processing of integers as well as the magnitude processing of proportions,

8 irrespective of symbolic or non-symbolic presentation format (for a brief overview on the

9 processing of symbolic and non-symbolic proportions, see Jacob, Vallentin, \& Nieder, 2012).

10 Yet, previous studies on the processing of proportions mainly focused on the processing of

11 their magnitude in the IPS. However, recent research showed that the intraparietal cortex hosts

12 other (related), more domain-general functions (e.g., Knops, Thirion, Hubbard, Michel, \&

13 Dehaene, 2009; Simon et al., 2002). This is in line with arguments from an evolutionary point of

14 view that number processing co-opts brain regions that support these related functions rather than

15 relying on a specifically dedicated brain region (Dehaene and Cohen, 2007; Knops et al., 2009). In

16 the following, number unspecific processes subserved by the intraparietal cortex will be briefly

17 summarized.

\subsection{Sensory, motor, and cognitive processes of the IPS}

The parietal cortex is associated with a wide range of sensory, motor, and cognitive functions

21 (Critchley, 1953). Because of its complex and multimodal responses, the parietal cortex is

22 considered as part of the "tertiary association cortex" (Culham and Kanwisher, 2001). In particular,

\footnotetext{
${ }^{1}$ The base-10 place-value structure is based on the position of each digit which determines the numerical value within the respective digit string without proportional aspects (i.e., base-10 place-value structure: e.g., $35.4=3 \times 10^{1}+5 \times$ $10^{0}+4 \times 10^{-1}$; Huber et al., 2014b).
} 
1 a variety of visuospatial tasks has been found to activate the parietal cortex such as hand reaching,

2 grasping, saccades, attention orienting, mental rotation, and spatial working memory as well as

3 guidance of actions yield activation (Culham and Kanwisher, 2001; Simon et al., 2002). These

4 cognitive functions partly share processes across domains, and thus, show overlapping activation

5 in the parietal cortex while smaller sub-regions exhibit domain-specific activations (Humphreys

6 and Lambon Ralph, 2015).

7 In particular, IPS and superior parietal lobule (SPL) are involved in a variety of tasks

8 including top-down attention, numerical calculation, executive semantics, phonological tasks, and

9 tool-praxis decisions (Humphreys and Lambon Ralph, 2015). Furthermore, IPS and SPL are

10 specifically involved in the storage and manipulation of items held in working memory during

11 goal-directed and executively demanding tasks (Humphreys and Lambon Ralph, 2015; Jonides et

12 al., 1998). As these tasks are non-automatic, goal-directed and highly demanding, it was assumed

13 that these brain regions support a general top-down processing system as part of a fronto-parietal

14 executive system across domains (Cabeza, 2008; Corbetta and Shulman, 2002; Humphreys and

15 Lambon Ralph, 2017, 2015).

16 Visual attention processes and reorienting spatial attention are widely considered to show a

17 right hemispheric predominance (Arrington et al., 2000; Corbetta et al., 2000; Thiel et al., 2004).

18 Also neuropsychological studies in patients with parietal lesions in the right hemisphere found

19 deficits in attentional processes (Becker and Karnath, 2007; Heilman et al., 1985; Sturm et al.,

20 2004), while motor attention has been shown to be linked to the left parietal cortex (Rushworth et

21 al., 2003, 2001).

22 Additionally, the IPS is involved in suppression of task-irrelevant distractors (Wojciulik and

23 Kanwisher, 1999) and response-selection processes (Göbel et al., 2004). Thus, in the parietal lobes

24 processes are subserved, which are not specific for magnitude processing itself, but are necessary 
1 for or associated with the processing of numbers such as top-down attention, spatial representation,

2 working memory, eye movements, and the guidance of actions (Culham and Kanwisher, 2001;

3 Humphreys and Lambon Ralph, 2015).

4 Thus, previous research indicated that the IPS hosts a domain-general multi-demand system

5 involving a variety of sensory, motor, and cognitive processes ranging from attentional processes

6 to mental navigation as well as from pointing and grasping to number processing (e.g., Humphreys

$7 \quad$ \& Lambon Ralph, 2015; Humphreys \& Ralph, 2017; Simon et al., 2002). Additionally, there is

8 evidence that these different goal-directed processes interact as they all require a domain-general

9 executive system (e.g., Hubbard, Piazza, Pinel, \& Dehaene, 2005; Humphreys \& Lambon Ralph,

10 2015). For instance, Knops and colleagues (2009) showed that brain circuits associated with spatial

11 attention contribute to mental arithmetic. These results substantiate the notion that number

12 processing co-opts parietal circuits involved in low-level sensorimotor tasks such as spatial

13 attention and eye movements.

14 Furthermore, shared activation for grasping and pointing was observed in left IPS extending

15 into superior parietal lobule (SPL) and postcentral sulcus (Simon et al., 2002). These motor tasks

16 (i.e., grasping and pointing) also showed joint activation with visuospatial tasks such as saccades

17 and attention orienting in bilateral SPL (Simon et al., 2004, 2002). Interestingly, overlapping

18 activation was also observed with mental calculation, language, and saccades in the left posterior

19 segment of the IPS beneath the angular gyrus (Simon et al., 2002). Thus, number processing and

20 mental calculation does not only co-opt parietal circuits of low-level sensorimotor tasks, but also

21 shares brain areas with other higher cognitive functions such as language. Importantly, these

22 different cognitive processes share common features of executive demands (Humphreys and

23 Lambon Ralph, 2017). As such, the IPS is not a brain region specifically dedicated to numerical

24 cognition. Rather, the ability to process numbers and numerosities seems to involve number- 
1 unspecific processes such as top-down processes, spatial attention, working memory, as well as

2 executive processes which are hosted in an intraparietal executive system across domains with

3 more domain-specific areas surrounding it (Humphreys and Lambon Ralph, 2017).

4 symbolic formats might be required besides domain-specific numerical processes (e.g.,

\subsection{The present study}

Previous research focused either on the magnitude processing of symbolic (DeWolf et al., 2016; Ischebeck et al., 2009a; Jacob and Nieder, 2009b) or non-symbolic proportions (Jacob and Nieder, 2009a) or on the question whether symbolic and non-symbolic proportions share an intraparietal neural correlate for overall magnitude processing, irrespective of presentation format (Mock et al., 2018). However, it has not yet been investigated whether there is a joint neural correlate in intraparietal areas associated with above described domain-specific numerical and domain-general cognitive processes. A closer look at the results of our latest study (Mock et al., 2018) provides an indication that there may be such shared intraparietal processes not necessarily specific to overall magnitude processing: the shared neural correlate in the right IPS for overall magnitude processing of symbolic and non-symbolic proportions as reflected by the numerical distance effect was rather small ( $k=46$ voxels; Mock et al., 2018). Typically, activated clusters in IPS are considerably larger for processing natural numbers or proportions, irrespective of presentation format (e.g., Arsalidou \& Taylor, 2011 for a review and meta-analysis). Thus, these larger clusters might reflect the involvement of further cognitive processes complementing magnitude processing. In fact, to operate on magnitude information of proportions (e.g., fractions, decimals, pie charts, and dot patterns), other rather domain-general cognitive processes such as attention, eye movements during the comparison, working memory, and mental rotation in nonmagnitude). Furthermore, even though overall magnitude processing of symbolic and non- 
1 symbolic proportions elicited joint activation in the right IPS (Mock et al., 2018), it is still unclear

2 whether the underlying neural processes to operate on this relative magnitude information are also

3 shared, or at least similar. Another important question we tackle in the present study is how bipartite

4 part-whole relations (e.g., fractions, pie charts, and dot patterns only) are processed at the neural

5 level. The bipartite structure of these presentation formats requires relating their components (e.g.,

6 denominator and numerator of a fraction) to infer overall magnitude information, which may

7 require additional domain-general cognitive processes to determine part-whole relations.

8 To pursue this issue, the present study took a two-step procedure. In a first step, we aimed at

9 investigating whether there is a joint neural correlate for domain-specific numerical and domain-

10 general processes in proportion processing at the whole-brain level. In a second step, we

11 investigated the processing of part-whole relation in more detail by contrasting conditions requiring

12 processing of part-whole relations (i.e., fractions, dot patterns, and pie charts) to decimals, which

13 do not require part-whole but base-10 processing. Additionally, based on the results of the first

14 step, we also pursued the question whether possibly shared activation patterns also indicate shared

15 underlying neural processes in the parietal cortex. It is important to note that the current work and

16 Mock et al. (2018) report on complementary aspects of the same dataset and that the current results

17 reflect analyses of both domain-specific numerical as well as domain-general processes as

18 compared to the specific processing of overall proportion magnitude in the IPS (as reflected by the

19 numerical distance effect) as evaluated by Mock et al. (2018).

20 We employed four different magnitude comparison tasks with symbolic (e.g., fractions and

21 decimals) and non-symbolic proportions (e.g., dot patterns and pie charts), respectively. As

22 previous studies showed an involvement of bilateral parietal regions and the prefrontal cortex

23 (PFC) during processing of symbolic and non-symbolic proportions, we expected a shared neural

24 correlate for all four presentation formats in this fronto-parietal network including IPS. First, we 
1 identified joint intraparietal activation using a conjunction analysis on the whole-brain level.

2 Second, and most importantly, as DeWolf and colleagues (2016) found that symbolic part-whole

3 relations (e.g., fractions) yielded an activation pattern distinct from that observed for numbers

4 complying with the base-10 structure, we examined the contrast between proportions reflecting

5 bipartite part-whole relations (i.e., fractions, dot patterns, and pie charts) and decimals on a whole-

6 brain level. This way, we were able to evaluate activation specific to part-whole processing without

7 the interference of base-10 processing. Furthermore, in doing so we extended the scope of previous

8 research (DeWolf et al., 2016) by including non-symbolic presentation formats.

9 To further evaluate proportion processing specifically in the parietal cortex, the resulting

10 clusters of the conjunction analysis were taken as regions of interest (ROIs) for which the respective

11 BOLD signal change was evaluated. Finally, possible differences between processing part-whole

12 relations versus decimals were evaluated by a representational similarity analysis (RSA)

13 differentiating the similarity of the intraparietal BOLD response for the different presentation

14 formats in the bilateral IPL.

\section{2. Results}

\section{$\underline{2.1 \text { Behavioral results }}$}

18 Mean speed of participants in the four conditions for dot patterns, fractions, pie charts, and

19 decimals, respectively, was: $M_{\text {dots }}=0.57(S E=0.04)$ items $/ \mathrm{sec}, M_{\text {fractions }}=0.57(S E=0.03)$

$20 \mathrm{items} / \mathrm{sec}, M_{\text {pies }}=0.85(S E=0.04) \mathrm{items} / \mathrm{sec}$, and $M_{\text {decimals }}=1.10(S E=0.03) \mathrm{items} / \mathrm{sec}$.

21 In the next step, a univariate ANOVA with speed as the dependent variable and presentation

22 format (dot patterns, pie charts, fractions, and decimals) as a fixed factor was conducted to compare

23 the effect of presentation format on speed. The main effect was highly significant [presentation

24 format: $F(3,104)=48,57, p<.001, \eta_{G}^{2}=.584$ ], indicating that participants' speed differed between 
1 presentation formats. Post-hoc comparisons revealed that except for the difference between dot

2 patterns and fractions $(p=1)$ speed differed significantly from each other in all presentation formats

3 (all $p<.001)$. Figure 1A gives an overview for speed data.

4 Moreover, mean accuracy in the four conditions for dot patterns, fractions, pie charts, and 5 decimals, respectively, were: $M_{\text {dots }}=70.7 \%(S E=2.75 \%), M_{\text {fractions }}=77.7 \%(S E=2.77 \%), M_{\text {pies }}=$

$691.0 \%(S E=0.9 \%)$, and $M_{\text {decimals }}=98.0 \%(S E=1.0 \%)$. Again, the univariate ANOVA revealed

7 significant main effect of presentation format $\left[F(3,104)=36.02, p<.001, \eta_{G}^{2}=.510\right]$. Post-hoc

8 comparisons indicated that the difference in accuracy was significant for all presentation formats

$9 \quad(p<.001)$ except the difference between pie charts and decimals $(p=.082)$ and between dot patterns

10 and fractions $(p=.090)$. Accuracy for the different presentation formats is shown in Figure $1 \mathrm{~B}$.

$>$ Insert Figure 1 here <

\section{$\underline{2.2 \text { Imaging results }}$}

\subsubsection{Whole-brain conjunction analysis}

16 The conjunction analysis across the four conditions (e.g., fractions, decimals, dot patterns,

17 pie charts) revealed significant joint activation in visual occipital areas (e.g., lingual gyrus, 18 calcarine gyrus, inferior occipital gyrus), bilateral thalamus and supplementary motor areas.

19 Importantly, joint activation was also found in bilateral inferior parietal cortex, close to its junction 20 with the IPS (see Table 1 and Figure 2). 


\section{> Insert Figure 4 here <}




\subsubsection{Representational similarity}

2 Descriptively, the resulting RSMs for left and right hemispheres were very similar (see Figure

3 5). We observed that the activation pattern for dot patterns was very similar to the one for fractions

$4 \quad(\mathrm{LH}: \operatorname{sim}=0.93 ; S D=0.07 ; \mathrm{RH}: \operatorname{sim}=0.92 ; S D=0.06)$ and pie charts $(\mathrm{LH}: \operatorname{sim}=0.88 ; S D=0.10$;

5 RH: $\operatorname{sim}=0.89 ; S D=0.09)$, whereas the activation pattern for decimals differed more from dot

6 patterns (LH: $\operatorname{sim}=0.77 ; S D=0.17 ; \mathrm{RH}: \operatorname{sim}=0.74 ; S D=0.16$ ). Furthermore, the activation

7 pattern for pie charts was more similar to the activation pattern for decimals (LH: $\operatorname{sim}=0.86 ; S D$

$8=0.13 ; \mathrm{RH}: \operatorname{sim}=0.83 ; S D=0.10)$. Moreover, the similarity of the activation patterns for pie charts

9 and fractions (LH: $\operatorname{sim}=0.84 ; S D=0.12 ; \mathrm{RH}: \operatorname{sim}=0.84 ; S D=0.08$ ) was in the same range as the

10 similarity between the one for pie charts and the other presentation formats. However, activation

11 patterns for decimals and fractions differed more strongly $(\mathrm{LH}: \operatorname{sim}=0.74 ; S D=0.19$; RH: $\operatorname{sim}=$

$120.70 ; S D=0.18)$.

$>$ Insert Figure 5 here <

The resulting dendrograms of the hierarchical cluster analysis for left and right hemisphere

17 are shown in Figure 6. Comparable to the RSM, it indicated that activation patterns for fractions,

18 dots, and pie charts were very similar, whereas activation patterns of decimals differed from the 19 other presentation formats.

\section{3. Discussion}


The present study aimed at investigating whether there is a shared neural substrate in the IPL

2 for domain-specific numerical as well as domain-general aspects of processing proportions beyond

3 processing overall magnitude (as reflected by the numerical distance effect, cf. Mock et al., 2018).

4 It is important to note that we already reported complementary aspects specific to overall

5 magnitude processing of this dataset in Mock et al. (2018). Here, we aimed at evaluating the

6 processing of proportions more broadly and part-whole relations in particular. Additionally, we

7 were interested in whether shared neural activation patterns in the parietal cortex may also indicate

8 shared underlying neural processes across different notations. To explore the cognitive demands

9 during proportion processing in bilateral IPL in more detail, we applied an analysis of the BOLD

10 signal change and a complementary RSA based on clusters of joint parietal activation identified in

11 our conjunction analysis. In the following, we will first discuss the joint neural substrate found for

12 processing proportions and part-whole relations in particular before we elaborate on our findings

13 on shared underlying neural processes within the IPL.

\section{$\underline{3.1 \text { A joint intraparietal neural substrate for processing of proportions }}$}

16 Our observation of joint bilateral IPL activation is in line with a recent meta-analysis by

17 Sokolowski and colleagues (2017) who reported joint activation in bilateral IPL for the processing

18 of symbolic and non-symbolic natural numbers. Importantly, our results extend this previous

19 research because we used symbolic and non-symbolic proportions instead of integers and

20 numerosities only. This substantiates the idea of IPL to be crucially involved in number processing

21 in general.

22 Yet, the joint neural correlate found in our data may not exclusively reflect number magnitude

23 processing (Sokolowski et al., 2017). On the other hand, we also do not wish to claim that the joint

24 activation found in bilateral IPL exclusively reflects domain-general processes without any aspect 
1 of magnitude processing. Instead, the observed IPL activation may well reflect processing and

2 operating on number magnitude information. However, when investigating intraparietal processing

3 specific to overall magnitude processing within the very same data set in a previous study (Mock

4 et al., 2018, reflected by the numerical distance effect), we observed joint activation for overall

5 magnitude processing of these presentation formats in a small cluster in right IPS only (MNI: 31 ,

$6-60,60 ; k=46$ voxels). Thus, the core area for the processing of overall relative magnitude differed

7 from the region we found in the present study (see also Figure 2). Therefore, we suggest that the

8 neural overlap found in the present study might rather reflect shared task demands for all four

9 presentation formats such as visuo-spatial and top-down attention (Humphreys and Lambon Ralph,

10 2017), estimation, summation, and calculation processes (Castelli et al., 2006; Holloway et al.,

11 2010; Piazza et al., 2006; Venkatraman et al., 2005), working memory during maintenance and

12 manipulation of the to-be-compared items (Humphreys and Lambon Ralph, 2015; Jonides et al.,

13 1998), eye movements (Knops et al., 2009; Simon et al., 2004, 2002), or response-selection (Göbel

14 et al., 2004).

15 Although symbolic and non-symbolic proportions used in this study were conceptually

16 distinct (part-whole vs. base-10), we nevertheless found a joint neural substrate in the parietal

17 cortex indicating shared processes. Unfortunately, we are not able to further distinguish the

18 processes involved on the basis of our design. However, we speculate that these processes might

19 include domain-general visuo-spatial and top-down attentional processes, and working memory

20 (Culham and Kanwisher, 2001; Göbel et al., 2004; Humphreys and Lambon Ralph, 2017, 2015;

21 Simon et al., 2002) but also more domain-specific estimation, summation, and calculation

22 processes (Castelli et al., 2006; Holloway et al., 2010; Piazza et al., 2006; Venkatraman et al.,

23 2005) necessary to compute the relative magnitude of the given proportions. This is in line with

24 the assumption of Humphreys and Lambon Ralph $(2015 ; 2017)$ who proposed a domain-general 
1 executive system in the IPS and SPL for shared non-automatic top-down processes during

2 demanding, goal-directed tasks. Overall proportion magnitude, however, may be processed in the

3 right IPS (Mock et al., 2018), which might correspond to a more domain-specific subregion close

4 to this domain-general executive system in a nearby, but different brain area.

5 As working memory processes are also associated with frontal brain areas, previous studies

6 additionally reported activation in PFC during processing symbolic fractions and non-symbolic

7 proportions (Ischebeck et al., 2009a; Jacob and Nieder, 2009a, 2009b). Hence, we also expected a

8 joint fronto-parietal network being involved in the processing of symbolic and non-symbolic

9 proportions. However, we only observed activation in the dorsolateral PFC for the processing of

10 fractions, dot patterns and pie charts, but not for the processing of decimals (see Appendix for the

11 whole-brain analysis of the four presentation formats against baseline, respectively). As such, the

12 results of our conjunction analysis did not reveal joint prefrontal activation. This suggests that

13 fractions and non-symbolic proportions such as pie charts and dot patterns may be processed

14 differently from decimals, as only the former activated a fronto-parietal network whereas the latter

15 did not (see Appendix; for similar results on differences in processing fractions and decimals, see

16 DeWolf et al., 2016).

\subsection{Neural processing of part-whole relations}

19 Fractions, dot patterns, and pie charts reflect bipartite part-whole relations. In particular, the

20 bipartite structure of these presentation formats requires relating their two parts to infer the

21 respective relative magnitude information. That is, the relation between the two proportional

22 components determines overall relative magnitude. In contrast, decimals do not require these

23 relating processes or additional computational steps and simply reflect their magnitudes based on

24 their base-10 structure. To disentangle part-whole from base-10 processing, we evaluated the 
1 contrast between part-whole proportions and decimals. Thereby, we were able to investigate part-

2 whole relation processing without interfering symbol-to-referent mapping of base-10 notations.

3 We found large activated clusters in bilateral IPL extending to bilateral occipital gyrus,

4 bilateral SMA, bilateral insula, as well as clusters in bilateral middle and inferior frontal gyrus.

5 Parts of the activation found in bilateral IPL (i.e., right IPS) have been associated with magnitude-

6 specific processing of proportions previously (Mock et al., 2018). However, as these brain regions

7 also seem to host an intraparietal domain-independent system of executive processes, the observed

8 activation might also indicate the involvement of top-down processes, spatial attention, working

9 memory, and other executive processes (e.g., Fias et al., 2013; Humphreys \& Lambon Ralph,

10 2017). The activation of bilateral inferior frontal gyri also supports the idea of an involvement of

11 top-down and working memory processes. Activation in these brain areas was associated with 12 visual working memory (e.g., Song and Jiang, 2006), higher cognitive monitoring such as

13 choosing, comparing, or judging and manipulation of information (e.g., Christoff et al., 2000;

14 Ranganath et al., 2003), procedural complexity (e.g., Delazer et al., 2003; Simon et al., 2002), as

15 well as attentional processes (e.g., Ischebeck et al., 2009b). Furthermore, the inferior frontal gyri

16 also seem to underlie strategy choice and planning in mathematical processes (e.g., Arsalidou and

17 Taylor, 2011; Dehaene and Cohen, 1997). Additionally, bilateral insula was associated with the

18 execution of responses (e.g., Huettel et al., 2001) and goal-directed motivated behavior (e.g., Uddin

$19 \&$ Menon, 2009). Thus, the broad activation of a fronto-parietal pattern of brain areas may suggest

20 the involvement of domain-general cognitive processes such as working memory, top-down

21 attentional and executive processes, as well as strategy choice that go beyond pure magnitude

22 processing.

23 Interestingly, joint activation of dorsolateral frontal cortex including inferior and middle

24 frontal gyrus, insula, precentral gyrus, and SMA, as well as areas in and around the IPS was 
1 interpreted to reflect a so-called multiple demand system (MD, see Duncan, 2010; Fedorenko et

2 al., 2013) or an extrinsic mode network (EMN, see Hugdahl and colleagues, 2015). The MD system

3 is associated with diverse cognitive and executive control demands including perception, response

4 selection, language, memory processes, problem solving, and task novelty independent of the

5 actual task at hand (Duncan, 2013, 2010; Fedorenko et al., 2013). The EMN extends the concept

6 of the MD suggesting a common activation pattern structure and up-regulating during task

7 processing, independent of the specific cognitive task at hand (Hugdahl et al., 2015). For instance,

8 EMN was shown to be activated by distinct cognitive tasks (e.g., spatial working memory, response

9 inhibition, impulse control, executive function, mental rotation, and arithmetic) and responds to

10 focused attention, goal maintenance, strategy selection, working memory and performance

11 monitoring (Fedorenko et al., 2013; Hugdahl et al., 2015). Hence, brain areas associated with MD

12 and the EMN show a broad functional generality.

13 Importantly, the brain regions we found for part-whole relation processing are strikingly

14 similar to the ones comprised by the MD and the EMN. This further supports the notion of domain-

15 general processes being involved in the processing of part-whole relations, not only on the whole-

16 brain level but also specifically in parietal brain regions. It is important to note, however, that we

17 do not suggest that part-whole relations are exclusively processed by domain-general networks.

18 Instead, like all human cognition, this processing is accomplished by a combination of domain-

19 specific regions like the parts of the IPS for number magnitude processing (Mock et al., 2018) and

20 domain-general cognitive and neural mechanisms (Duncan, 2013; Fedorenko et al., 2013; Hugdahl

21 et al., 2015). Thus, for the case of processing part-whole relations MD and EMN are complemented

22 by highly specialized brain regions.

23 However, the question remains why we find MD and EMN activation for part-whole relation

24 processing, but not for processing proportions in general including decimals. Hugdahl and 
1 colleagues (2015) argued that the EMN is sensitive to learning and experience, which means that

2 overlearned tasks do not activate the EMN (Hugdahl et al., 2015). As magnitude processing of

3 decimals is very similar to the processing of other multi-digit numbers (when skipping the leading

4 0) adults are confronted permanently with in everyday life, we suggest that decimals may be

5 overlearned as compared to symbolic or non-symbolic proportions. Hugdahl and colleagues (2015)

6 further suggested that the EMN follows a threshold effect which varies depending on task novelty

7 (Hugdahl et al., 2015). This way, the absence of MD and EMN in our conjunction analysis can be

8 explained by the fact that processing of decimals reduces the experience of novelty, and thus, down-

9 regulates the EMN. In contrast, when task novelty is high, which was apparently the case in the

10 part-whole relation tasks, EMN is up-regulated complementing highly domain-specific brain

11 mechanisms.

\subsection{Cognitive demands during proportion processing in bilateral IPL}

14 When analyzing BOLD signal change in the bilateral IPL ROIs identified by the conjunction

15 analysis, we only found a main effect for the four presentation formats. There was no difference

16 between hemispheres and also no interaction although left parietal cortex was recently suggested

17 to be associated rather with the processing of symbolic numbers whereas right parietal cortex with

18 the processing of non-symbolic numerosities (Cappelletti et al., 2010, 2009; Holloway et al., 2010;

19 Sokolowski et al., 2017). However, previous research on this lateralization mainly focused on

20 number magnitude processing, while we investigated proportion processing beyond overall

21 magnitude processing. This might also indicate that the BOLD signal change found in our data

22 rather reflects other cognitive processes necessary for the processing of symbolic and non-symbolic

23 proportions than just overall magnitude processing. 
The highest signal change was found for both non-symbolic presentation formats (e.g., dot

2 patterns and pie charts), probably indicating higher demands on visuo-spatial attention as well as

3 maintenance and manipulation of the respective items in working memory. In particular, the

4 processing of proportional dot patterns might require more eye movements between the dots for

5 individuation and summation of non-symbolic items (Holloway et al., 2010) whereas the

6 processing of pie charts might rather involve mental rotation strategies (Alivisatos and Petrides,

7 1997; Jordan et al., 2001). Regardless of whether summation or mental rotation strategies are used

8 to finally operate on the magnitude of those non-symbolic formats, the task is highly demanding

9 and visuo-spatial attention is highly required. This assumption is further substantiated by additional

10 activation observed in bilateral frontal eye fields (FEF) and posterior superior parietal lobule

11 (PSPL) for both of these non-symbolic presentation formats as these regions are typically

12 associated with eye movements (see Appendix A3 and A4; Knops et al., 2009). Additionally,

13 activation in PFC reflect the involvement of executive control, working memory and attentional

14 processes sending top-down signals to IPL (Humphreys and Lambon Ralph, 2015).

15 Furthermore, signal change for fractions was higher compared to decimals. This might

16 indicate higher attentional loads as well as working memory demands during additional

17 computational steps to access overall magnitude information of fractions (Mock et al., 2018).

18 Moreover, eye movements between the two fractions or between their numerators and

19 denominators might lead to higher visuo-spatial attention loads. In fact, for the processing of

20 fractions we also observed additional activation in FEF and PSPL which are typically associated

21 with eye movements (Knops et al., 2009) and prefrontal activation reflecting top-down attentional

22 and working memory processes (see Appendix A1; Humphreys \& Lambon Ralph, 2015).

23 In contrast, no additional computations and only low attentional demands are required while 24 processing decimals. Rather, a simple symbol-to-referent mapping might seem plausible for 
1 decimals as they directly reflect number magnitudes (Holloway et al., 2010). This idea is

2 corroborated by activation in areas subserving these processes such as left angular gyrus and

3 superior and middle temporal gyrus, which can be observed for decimals using a slightly less

4 conservative correction for the fMRI data $(p<.001$, uncorrected) than reported in the Appendix

$5 \mathrm{~A} 2(p<.05$, FWE-corrected). In particular, these brain regions are rather associated with bottom-

6 up and automatic processes which further supports the assumption of a direct symbol-to-referent

7 mapping (Humphreys and Lambon Ralph, 2015). Thus, in contrast to the bipartite structure of the

8 other presentation formats decimals do not seem to reflect proportional aspects in the same way.

9 Importantly, activation in parietal brain regions was previously associated with tasks

10 requiring additional attention due to higher levels of difficulty (Culham et al., 1998; Culham and

11 Kanwisher, 2001; Humphreys and Lambon Ralph, 2017; Shuman and Kanwisher, 2004). Thus,

12 parietal activation during numerical tasks might also be driven by task difficulty to some degree.

13 However, in the present study the signal change induced by proportion processing did not exactly

14 resemble the pattern found in the behavioral data. That is, the difficulty pattern found in speed and

15 accuracy differed from the pattern observed for signal change in the bilateral IPL. Speed was lowest

16 for fractions and dot patterns. Yet, comparing pie charts led to faster responses, while participants

17 achieved highest speed when comparing decimals. Additionally, accuracy in our data was highest

18 for decimals and pie charts, whereas accuracy for fractions and dot patterns was similarly low. As

19 increasing response times and error rates might indicate influences of task difficulty, these

20 behavioral results seem to reflect the difficulty in operating on magnitude information between the

21 different presentation formats. However, the signal change analysis revealed a different pattern.

22 Furthermore, previous studies found activations in the intraparietal cortex for either passive

23 listening to number words (Klein et al., 2010) or passive viewing of symbolic numbers versus

24 letters and colors (Eger et al., 2003). This indicates that intraparietal activations can be associated, 
1 at least partially, with number processing independent from difficulty level. Thus, our results

2 cannot be attributed to differences in difficulty exclusively.

\subsection{Shared underlying neural processes of symbolic and non-symbolic proportions}

5 Although fractions, dot patterns, pie charts, and decimals reflect conceptually different properties

6 (part-whole vs. base-10), they activated a joint neural correlate as reflected by the conjunction

7 analysis. However, a joint neural substrate does not necessarily implicate one common underlying

8 process. Therefore, we conducted an RSA to gain more fine-grained results because this analysis

9 evaluates similarities in the activation patterns of the four presentation formats.

10 The RSA revealed that the patterns of neural activations elicited by the part-whole relations (i.e.,

11 fractions, dot patterns, and pie charts) were very similar. That is, voxels in bilateral IPL responded

12 very similarly when processing symbolic fractions as well as non-symbolic dot patterns and pie

13 charts.

14 Thereby, our results indicate a dissociation between the patterns of neural activation elicited by

15 decimals and the other presentation formats which further substantiates our contrast analysis.

16 Furthermore, this is in line with the results of DeWolf and colleagues (2016) who observed that the

17 activation pattern for fractions differed systematically from that of decimals and integers within

18 the parietal cortex. Whereas the latter two number formats were hardly distinguishable, DeWolf

19 and colleagues (2016) were able to clearly differentiate activations for fractions. Thus, the major

20 notations for symbolic proportions (i.e., fractions and decimals) seem to draw on distinct neural

21 processes even when they express the same magnitude (e.g., $2 / 5$ and 0.4). The present results

22 indicate that fractions share an underlying neural process with proportional dot patterns and pie

23 charts, whereas the neural processing of decimals differs. A plausible explanation for our finding

24 is that the underlying neural processing of fractions, dot patterns and pie charts reflects the bipartite 
1 structure of proportional part-whole relations. In contrast, decimals simply and directly reflect

2 number magnitudes in a base-10 notation (e.g., see Huber et al., 2014b) and do not require the

3 additional processing of proportional aspects. Thus, the structure of proportions (part-whole vs.

4 base-10) seems to determine their underlying neural processing. Depending on the structure task

5 demands might differ which, in turn, seems to change the respective activation patterns. This is in

6 line with Humphreys and Lambon Ralph (2015) who suggested that parietal organization is not

7 split by domain-specific tasks but rather meets the requirements of demanding tasks by adjusting

8 respective neurocomputations. While performing a task, the respective activation pattern within

9 parietal cortex might change due to different task demands (Humphreys and Lambon Ralph, 2015;

10 see also Hugdahl et al., 2015 for a similar threshold effect in the EMN). Relating this to our data,

11 pie charts, dot patterns, and fractions might have elicited similar neurocomputations within the IPL

12 because of their structural similarity (i.e., bipartite part-whole relation), which, in turn, is reflected

13 by the reported RSA pattern. In contrast, neurocomputations required for processing decimals on

14 the neural level might differ as they do not reflect this bipartite part-whole relation.

\section{$\underline{3.6 \text { Limitations }}$}

17 We are well aware that the current study is only a first step towards a more comprehensive 18 understanding of the underlying processes in proportion processing. Thus, several aspects need to

19 be considered when interpreting the results.

20 First, the design of the present study does not allow to further differentiate between domain-

21 specific and domain-general mechanisms involved in proportion processing as the task at hand was

22 a magnitude comparison task. However, in our view, activation patterns found for proportion

23 processing in the present study do not reflect magnitude processing exclusively because we

24 observed a core area for overall magnitude processing reflected by the numerical distance effect 
1 exclusively in the right IPS for the same data set (Mock et al., 2018). Thus, although magnitude

2 information might be processed as well in the present study, additional activation found here might

3 reflect the additional involvement of more domain-general processes associated with proportion

4 processing. Although our interpretation has to remain speculative, our findings nevertheless fit well

5 with the literature. Yet, to disentangle this issue, future research is needed in which it would be

6 desirable to specify the suggested domain-general processes.

7 Second, it needs to be noted that the pattern of our RSA might be influenced by task difficulty.

8 In fact, participants responded more accurate and faster to decimals. In turn, they spent more time

9 viewing the scrambled mask consisting of visual noise than doing the actual proportion magnitude

10 comparison as compared to the other presentation formats. Furthermore, as we used a block design

11 attentional demands might be different between an easy block (e.g., comparing decimals) and a

12 more difficult block (e.g., comparing dot patterns). In turn, this might have led to lower correlations

13 in the RSA. However, usually influences of task difficulty are also reflected in the amplitude of

14 fMRI signal change (Culham et al., 2001; Ress et al., 2000). In line with above argument, signal

15 change was lowest for decimals. Nevertheless, the pattern found for signal change associated with

16 the other presentation formats differed from the pattern found in the RSA as the amplitude in signal

17 change was highest for the two non-symbolic formats. Thus, while we cannot fully exclude

18 influences of task difficulty in the RSA pattern, we suggest that the RSA does at least not

19 exclusively reflect task difficulty.

\section{4. Conclusion}

22 Proportion processing beyond overall magnitude processing elicited activation in frontal,

23 parietal and occipital brain regions including bilateral IPL. However, observed activation in

24 bilateral IPL, an area typically associated with number magnitude processing, did not reflect 
1 processing of overall proportion magnitude in our study. In fact, a shared neural substrate for the

2 processing of abstract relative magnitude was observed in a nearby, but different brain area (i.e.,

3 right IPS) in a different analysis on the very same data set (Mock et al., 2018; see also Figure 2).

4 Thus, the activation found in the present study does not necessarily reflect overall magnitude

5 processing. Nevertheless, the shared neural correlate for proportion processing indicates common

6 cognitive processes in a top-down executive parietal system. These processes might include visuo-

7 spatial and top-down attention, estimation and summation processes, working memory, response-

8 selection, and additional part-whole computations in the bilateral IPL for computing actual relative

9 magnitude as well as relating and integrating the to-be-compared proportions.

10 Investigating processing of part-whole relations, we found wide-spread activation in fronto-

11 parietal brain areas. This substantiates the involvement of domain-general cognitive processes in

12 part-whole processing such as working memory, top-down attentional and executive processes, as

13 well as strategy choice that go beyond magnitude processing. Thus, processing part-whole relations

14 seems to be accomplished as an interplay of domain-specific (Mock et al., 2018) and domain-

15 general cognitive and neural processes (Duncan, 2013; Fedorenko et al., 2013; Hugdahl et al.,

16 2015) that complement each other.

17 The dissociation between part-whole relations (i.e., fractions, dot patterns, and pie charts) and

18 decimals was substantiated by an additional RSA. Thus, although processing fractions, decimals,

19 dot patterns, and pie charts elicited joint neural activation, the underlying neural processes seemed

20 to be different depending on the structure of the respective proportion (i.e., part-whole vs. base-

21 10). Thus, underlying structural differences of the notations seem to lead to distinct underlying

22 neural processes.

24 5. Materials and Methods 


\section{$\underline{5.1 \text { Participants }}$}

Participants were 24 right-handed university students who participated voluntarily (13

3 females, mean age $=23.2$ years; $S D=2.99$ years). Participants gave their written consent according

4 to the protocol of the local Ethics Committee of the Medical Faculty of the University of Tuebingen

5 after being informed about the experimental procedure. All participants reported no previous

6 history of neurological or psychiatric disorders and normal or corrected to normal vision.

7 Participants received monetary compensation.

\subsection{Procedure}

10 Four different presentation formats of proportions were used in this study: fractions,

11 decimals, pie charts, and dot patterns. Proportion processing was investigated by means of blocked

12 magnitude comparison tasks (i.e., fractions, decimals, pie charts, and dot patterns). Proportions

13 were presented pseudo-randomly in 24 blocks (six blocks per condition) consisting of one practice

14 trial and four critical trials (120 trails in total: 24 practice and 96 experimental trials).

15 At the beginning of each block, a cue was presented for $500 \mathrm{~ms}$ indicating which proportion

16 type had to be compared in the next five trials. The cue was the fraction $1 / 4$ shown in the respective

17 presentation formats in the center of the screen against a grey background. Afterwards, a black

18 screen was presented for $4,000 \mathrm{~ms}$. Subsequently, trials started with a black fixation cross against

19 a grey background for $500 \mathrm{~ms}$, followed by the presentation of the to-be-compared proportion

20 stimuli for up to 5,000 ms. Participants had to respond within this time frame by pressing one of

21 two MRI compatible response buttons with either their left or right thumb indicating that either the

22 left or the right proportion was larger, respectively. When participants responded faster than the

23 given 5,000 ms, a mask consisting of visual noise (blue, yellow, and grey pixels) was presented for 
1 the remaining time. Then the next trial started. The procedure of the beginning of a block is shown

2 in Figure 7. At the end of each block, a black screen was presented for 6,000 ms.

3

4

5

6

9 from 0.22 to 0.89 .

11 formats. Numerators of the fractions ranged from 1 to 8 and denominators from 2 to 9. Fractions

12 were constructed such that in half of the items the comparison of numerators and denominators

13 was either congruent or incongruent with the comparison of overall fraction magnitude. For

14 congruent trials, separate comparisons of numerator and denominator magnitudes yielded the same

15 answer as the comparison of the overall magnitudes of fraction pairs (e.g., $1 / 6<3 / 8$ with $1<3$ and

$166<8$ ). In contrast, separate comparisons of numerator and denominator magnitudes yielded

17 opposing answers as compared to the overall magnitude of the fractions in incongruent trials (e.g.,

$184 / 7<2 / 3$, but $4>2$ and $7>3$ ). Thus, participants were not able to solve the task correctly, when

19 relying on the magnitude of numerators or denominators only. We then constructed decimals by

20 dividing numerators by denominators and rounding the result to two digits after the decimal point.

21 Decimals and fractions were presented in blue (font type: Arial; font size: 80; RGB-values: 53, 85,

22 204) against a grey background (RGB-values: 204, 204, 204). One fraction was located on the left

23 side (x/y-coordinates: $356 / 384$ px), whereas the other one was located on the right side (x/y-

24 coordinates: 668 / 384 px) of the screen (screen resolution: $1024 \times 768$ px). 
Dot patterns were drawn in the center of the left and the right side of the screen on an invisible

2 rectangular area of size $491 \times 363$ px. Location of dots varied randomly in these invisible

3 rectangular areas. Dot patterns were colored according to the fractions they denoted using blue

4 (same blue as for fractions) and yellow (RGB-values: 203, 187, 0) against a grey background (same

5 grey as for fractions and decimals). For instance, the dot pattern of 4/7 was drawn by coloring 4

6 dots in blue and 3 dots in yellow (and thus, 4 out of 7 dots were colored in blue). Diameter of dots

7 varied randomly from $21 \mathrm{px}$ to $98 \mathrm{px}$. To ensure that participants cannot rely on the proportion of

8 the sum of visual areas when comparing the dot patterns, the sum of yellow and blue areas of the

9 dots across the two dot patterns, which had to be compared, was equated.

10 Pie charts were drawn by dividing circles into pie segments according to the magnitude of

11 the respective fraction items using the same colors as for dot patterns. For instance, 4/7 was drawn

12 by coloring $4 / 7$ of the pie in blue and $3 / 7$ in yellow. The background color used was the same grey

13 as for the other presentation formats. The location of the yellow part varied pseudo-randomly. The

14 diameter of pies ranged from $95 \mathrm{px}$ to $289 \mathrm{px}$. To ensure that participants cannot select the larger

15 proportion by relying only on the visual area of pie segments, we varied the size of the circles such

16 that in half of the items the larger proportion was also larger in accordance to the visual area of the

17 yellow pie segment, whereas in the other half of the items it was smaller.

\section{$>$ Insert Figure 8 here $<$}

\section{4 fMRI data acquisition}

We acquired MRI data using a 3T Siemens Magnetom TrioTim MRI system (Siemens AG,

23 Erlangen, Germany). A high resolution T1-weighted anatomical scan (TR $=2300$ s, matrix $=256$

$24 \times 256,176$ slices, voxel size $=1.0 \times 1.0 \times 1.0 \mathrm{~mm}^{3} ; \mathrm{FOV}=256 \mathrm{~mm}^{2}, \mathrm{TE}=2.92 \mathrm{~ms}$; flip angle $=$ 
$18^{\circ}$ ) was run at the end of the experimental session. All functional measurements covered the whole

2 brain using standard echo-planar-imaging (EPI) sequences (TR =2,400 ms; $T E=30$ ms; flip angle $3=80^{\circ} ; \mathrm{FOV}=220 \mathrm{~mm}^{2}, 88 \times 88$ matrix; 42 slices, voxel size $\left.=2.5 \times 2.5 \times 3.0 \mathrm{~mm}^{3}, \mathrm{gap}=10 \%\right)$.

$4 \quad$ FMRI data was acquired in a single run. We included pauses between blocks each lasting for $56,000 \mathrm{~ms}$ and presenting a black screen after each block. Total scanning time was about 20 minutes.

6

$12(84,4 \%$ of all trials). time corrected, motion corrected, and realigned to each participant's mean image. No participant

22 had to be excluded due to head movements as motion parameters did not exceed $2.5 \mathrm{~mm}$ translation

23 in total and a head rotation of 1.5 degree in pitch, roll, and yaw in total. The mean image was co24 registered with the whole-brain volume. Then, imaging data was normalized into standard 
1 stereotaxic MNI space (Montreal Neurological Institute, McGill University, Montreal, Canada).

2 Images were resampled every $2.5 \mathrm{~mm}$ using 4th degree spline interpolation to obtain isovoxel and

3 then smoothed with a 6 mm full-width half-maximum (FWHM) Gaussian kernel to accommodate

4 inter-subject variation in brain anatomy and to increase signal-to-noise ratio in the images. To

5 remove low-frequency noise components the data were high-pass filtered (128s) and corrected for

6 autocorrelation assuming an $\mathrm{AR}(1)$ process.

7 The onsets of the four presentation formats (i.e., fractions, decimals, pie charts, dot patterns)

8 were entered as separate conditions in the GLM. Movement parameters estimated at the

9 realignment stage of preprocessing were included as covariates of no interest. Brain activity was

10 convolved over all experimental trials with the canonical haemodynamic response function (HRF)

11 as implemented in SPM12 and its time and dispersion derivatives.

12 We conducted a conjunction analysis in order to evaluate the notion of a joint neural substrate

13 of proportion processing (conjunction null; Nichols, Brett, Andersson, Wager, \& Poline, 2005). In

14 a second step, we compared activations for the bipartite part-whole relations (i.e., fractions, dot

15 patterns, and pie charts) to decimals to investigate brain activation based on mere part-whole

16 processing.

17 The SPM Anatomy Toolbox (Eickhoff et al., 2005), available for all published

18 cytoarchitectonic maps (www.fz-juelich.de/ime/spm_anatomy_toolbox), was used for anatomical

19 localization of effects where applicable. In areas not yet implemented, the anatomical automatic

20 labelling tool (AAL) in SPM12 (http://www.cyceron.fr/web/aal

21 anatomical_automatic_labeling.html) was used. For the whole brain analysis, activations were

22 thresholded at a whole-brain family-wise error corrected (FWE) $p$-value of $<.05$ with a cluster size

23 of $k=10$ voxels. 


\subsubsection{Signal change and representational similarity analysis}

For percent signal change and representational similarity analyses, we focused on the clusters found in bilateral IPL as a result of the conjunction analysis. Furthermore, we conducted the analyses for both hemispheres separately because it was found that symbolic and non-symbolic formats are processed using both overlapping and distinct neural substrates (Sokolowski et al., 2017). Besides overlapping processing in bilateral IPL, bilateral precuneus, left SPL and right SFG, symbolic number processing (Holloway et al., 2010) and mental calculation (Arsalidou and Taylor, 2011; Dehaene et al., 1999) were especially associated with activation in left IPS. In contrast, parts of right parietal cortex were rather found to be involved in (visuospatial) attentional processes (Arrington et al., 2000; Corbetta et al., 2000; Thiel et al., 2004), processing of non-symbolic numerosities (Holloway et al., 2010; Sokolowski et al., 2017), and magnitude processing (Mock et al., 2018; Mussolin et al., 2013; Piazza et al., 2007).

We further investigated the similarity of activation patterns in jointly activated clusters around the bilateral IPS across presentation formats suggesting similar neural representations. For this, we conducted a representational similarity analysis (RSA). The output of the RSA was a matrix (representational similarity matrix) which reflected pairwise correlations between all stimuli. The RSA was based on $\beta$ estimates of the voxels within jointly activated clusters in IPL. To obtain the representational similarity matrix, we ran Pearson correlations between the four presentation formats for each participant separately, scaled them into $[0,1]$ by computing the similarity value $\operatorname{sim}=(\mathrm{r}+1) / 2$, and then averaged similarity values across participants. The higher the value of similarity ( $\operatorname{sim})$, the more similar was the neural representation of the different presentation formats with 1 being most similar. Visualized similarity served to characterize the shared representation (Kriegeskorte et al., 2008). 
1 In course of the RSA, we further conducted an agglomerative hierarchical cluster analysis

2 based on the mean $z$-standardized $\beta$ estimates for the four different notation formats. In the

3 hierarchical cluster analysis, we used the Euclidean distance as distance measure and average

4 linkage clustering as linkage criterion with lower distance being more similar. 


\section{$1 \quad$ Funding}

2 Julia Mock was supported by the German Research Foundation (DFG) providing funding to Ulrike

3 Cress, Hans-Christoph Nuerk and Korbinian Moeller (CR-110/8-1) and to Korbinian Moeller and

4 Elise Klein (MO 2525/2-1). Stefan Huber, Johannes Bloechle and Julia Bahnmueller were supported

5 by the Leibniz-Competition Fund providing funding to Elise Klein. Korbinian Moeller is faculty

6 member and principal investigator, Elise Klein and Julia Bahnmueller are associated members at the

7 LEAD Graduate School [GSC1028] and Research Network funded by the Excellence Initiative of the

8 German federal and state governments. Elise Klein was supported by a Margarete von Wrangell

9 fellowship from the Ministry of Science, Research and the Arts Baden-Württemberg.

\section{Declarations of interest}

12 None.

\section{Authors' contributions}

15 EK and KM conceived the study. KM, EK, and SH participated in its design. JB and JB performed 16 data collection. JM and SH performed processing and statistical analyses. JM drafted the manuscript;

17 all other authors revised it critically. All authors contributed to the interpretation of the data. All 18 authors read and approved the final manuscript.

19 


\section{References}

2 Alivisatos, B., Petrides, M., 1997. Functional activation of the human brain during mental rotation. Neuropsychologia 35, 111-118. https://doi.org/10.1016/S0028-3932(96)00083-8

4 Arrington, C.M., Carr, T.H., Mayer, A.R., Rao, S.M., 2000. Neural mechanisms of visual attention: object-based selection of a region in space. J. Cogn. Neurosci. 12, 106-117.

6 Arsalidou, M., Taylor, M.J., 2011. Is 2+2=4? Meta-analyses of brain areas needed for numbers and 7 calculations. Neuroimage 54, 2382-2393. https://doi.org/10.1016/j.neuroimage.2010.10.009

8 Becker, E., Karnath, H.O., 2007. Incidence of visual extinction after left versus right hemisphere $9 \quad$ stroke. Stroke 38, 3172-3174.

10 Cabeza, R., 2008. Role of parietal regions in episodic memory retrieval: The dual attentional

11 processes hypothesis. Neuropsychologia 46, 1813-27.

12 https://doi.org/10.1016/j.neuropsychologia.2008.03.019

13 Cappelletti, M., Lee, H.L., Freeman, E.D., Price, C.J., 2010. The role of the right and left parietal

14 lobes in the conceptual processing of numbers. J. Cogn. Neurosci. 22, 331-346.

15 https://doi.org/10.1162/jocn.2009.21246

16 Cappelletti, M., Muggleton, N., Walsh, V., 2009. Quantity without numbers and numbers without 17 quantity in the parietal cortex. Neuroimage 46, 522-529.

18 https://doi.org/10.1016/j.neuroimage.2009.02.016

19 Castelli, F., Glaser, D.E., Butterworth, B., 2006. Discrete and analogue quantity processing in the

20 parietal lobe: A functional MRI study. Proc. Natl. Acad. Sci. 103, 4693-4698.

21 https://doi.org/10.1073/pnas.0600444103

22 Christoff, K., John, D., Gabriel, E., 2000. The frontopolar cortex and human cognition : Evidence 23 for a rostrocaudal hierarchical organization within the human prefrontal cortex 28, 168-186.

24 Cohen Kadosh, R., Cohen Kadosh, K., Schuhmann, T., Kaas, A., Goebel, R., Henik, A., Sack, A.T., 
2007. Virtual Dyscalculia Induced by Parietal-Lobe TMS Impairs Automatic Magnitude Processing. Curr. Biol. 17, 689-693. https://doi.org/10.1016/j.cub.2007.02.056

3 Corbetta, M., Kincade, J.M., Ollinger, J.M., McAvoy, M.P., Shulman, G.L., 2000. Voluntary

4 orienting is dissociated from target detection in human posterior parietal cortex. Nat. Neurosci. $5 \quad 3,292-297$.

6 Corbetta, M., Shulman, G.L., 2002. Control of goal-directed and stimulus-driven attention in the 7 brain. Nat. Rev. Neurosci. 3, 201-215. https://doi.org/10.1038/nrn755

8 Critchley, M., 1953. The parietal lobes. Williams and Wilkins, Oxford, England.

9 Culham, J.C., Brandt, S.A., Cavanagh, P., Kanwisher, N., Dale, A.M., Tootell, R.B., 1998. Cortical 10 fMRI activation produced by attentive tracking of moving targets. J. Neurophysiol. 80, 2657112670.

12 Culham, J.C., Cavanagh, P., Kanwisher, N.G., 2001. Attention response functions: Characterizing 13 brain areas using fMRI activation during parametric variations of attentional load. Neuron 32, 14 737-745. https://doi.org/10.1016/S0896-6273(01)00499-8

15 Culham, J.C., Kanwisher, N., 2001. Neuroimaging of cognitive functions in human parietal cortex. 16 Curr. Opin. Neurobiol. 11, 157-163.

17 Dehaene, S., Cohen, L., 2007. Cultural recycling of cortical maps. Neuron 56, 384-398.

18 https://doi.org/10.1016/j.neuron.2007.10.004

19 Dehaene, S., Cohen, L., 1997. Cerebral pathways for calculation: Double dissociation between rote

20 verbal and quantitative knowledge of arithmetic. Cortex 33, 219-250.

$21 \quad$ https://doi.org/10.1016/S0010-9452(08)70002-9

22 Dehaene, S., Spelke, E.S., Pinel, P., Stanescu, R., Tsivkin, S., 1999. Sources of mathematical 23 thinking: behavioral and brain-imaging evidence. Science (80-. ). 284, 970-974.

24 https://doi.org/10.1126/science.284.5416.970 
1 Delazer, M., Domahs, F., Bartha, L., Brenneis, C., Lochy, A., Trieb, T., Benke, T., 2003. Learning complex arithmetic — an fMRI study. Cogn. Brain Res. 18, 76-88. https://doi.org/https://doi.org/10.1016/j.cogbrainres.2003.09.005

DeWolf, M., Chiang, J.N., Bassok, M., Holyoak, K.J., Monti, M.M., 2016. Neural representations of magnitude for natural and rational numbers. Neuroimage 141, 304-312. https://doi.org/10.1016/j.neuroimage.2016.07.052

Duncan, J., 2013. The structure of cognition: attentional episodes in mind and brain. Neuron 80, 3550. https://doi.org/10.1016/j.neuron.2013.09.015

Duncan, J., 2010. The multiple-demand (MD) system of the primate brain: mental programs for intelligent behaviour. Trends Cogn. Sci. 14, 172-179. https://doi.org/10.1016/j.tics.2010.01.004

Eger, E., Sterzer, P., Russ, M.O., Giraud, A.L., Kleinschmidt, A., 2003. A supramodal number representation in human intraparietal cortex. Neuron 37, 719-725. https://doi.org/10.1016/S0896-6273(03)00036-9

Eickhoff, S.B., Stephan, K.E., Mohlberg, H., Grefkes, C., Fink, G.R., Amunts, K., Zilles, K., 2005. A new SPM toolbox for combining probabilistic cytoarchitectonic maps and functional imaging data. Neuroimage 25, 1325-1335.

Fedorenko, E., Duncan, J., Kanwisher, N., 2013. Broad domain generality in focal regions of frontal and parietal cortex. https://doi.org/10.1073/pnas.1315235110

Fias, W., Menon, V., Szucs, D., 2013. Multiple components of developmental dyscalculia. Trends Neurosci. Educ. 2, 43-47. https://doi.org/https://doi.org/10.1016/j.tine.2013.06.006

Göbel, S.M., Johansen-Berg, H., Behrens, T., Rushworth, M.F.S., 2004. Response-Selection-Related Parietal Activation during Number Comparison. J. Cogn. Neurosci. 16, 1536-1551. https://doi.org/10.1162/0898929042568442 
1 Grabner, R.H., Ansari, D., Koschutnig, K., Reishofer, G., Ebner, F., Neuper, C., 2009. To retrieve or to calculate? Left angular gyrus mediates the retrieval of arithmetic facts during problem solving. Neuropsychologia 47, 604-608.

Heilman, K.M., Watson, R., Valenstein, E., 1985. Neglect and related disorders, in: Heilman, K.M., Valenstein, E. (Eds.), Clinical Neuropsychology. Oxford; New York, pp. 243-293.

Holloway, I.D., Price, G.R., Ansari, D., 2010. Common and segregated neural pathways for the processing of symbolic and nonsymbolic numerical magnitude: an fMRI study. Neuroimage 49, 1006-1017.

Hubbard, E.M., Piazza, M., Pinel, P., Dehaene, S., 2005. Interactions between number and space in parietal cortex. Nat. Rev. Neurosci. 6, 435-448. https://doi.org/10.1038/nrn1684

Huber, S., Klein, E., Willmes, K., Nuerk, H.-C., Moeller, K., 2014. Decimal fraction representations are not distinct from natural number representations - evidence from a combined eye-tracking and computational modeling approach. Front. Hum. Neurosci. 8, 1-14. https://doi.org/10.3389/fnhum.2014.00172

Huettel, S.A., Güzeldere, G., McCarthy, G., 2001. Dissociating the neural mechanisms of visual attention in change detection using functional MRI. J. Cogn. Neurosci. 13, 1006-1018. https://doi.org/10.1162/089892901753165908

Hugdahl, K., Raichle, M.E., Mitra, A., Specht, K., 2015. On the existence of a generalized nonspecific task-dependent network. Front. Hum. Neurosci. 9, 430. https://doi.org/10.3389/fnhum.2015.00430

Humphreys, G.F., Lambon Ralph, M.A., 2017. Mapping domain-selective and counterpointed domain-general higher cognitive functions in the lateral parietal cortex: Evidence from fMRI comparisons of difficulty-varying semantic versus visuo-spatial tasks, and functional connectivity analyses. Cereb. Cortex 27, 4199-4212. https://doi.org/10.1093/cercor/bhx107 
1 Humphreys, G.F., Lambon Ralph, M.A., 2015. Fusion and fission of cognitive functions in the human parietal cortex. Cereb. Cortex 25, 3547-3560. https://doi.org/10.1093/cercor/bhu198

IMB Corp., 2011. IBM SPSS Statistics for Windows.

4 Ischebeck, A., Koschutnig, K., Reishofer, G., Butterworth, B., Neuper, C., Ebner, F., 2010. Processing fractions and proportions: An fMRI study. Int. J. Psychophysiol. 77, 227. https://doi.org/10.1016/j.ijpsycho.2010.06.325

Ischebeck, A., Schocke, M., Delazer, M., 2009a. The processing and representation of fractions within the brain. An fMRI investigation. Neuroimage 47, 403-413. https://doi.org/10.1016/j.neuroimage.2009.03.041

10 Ischebeck, A., Zamarian, L., Schocke, M., Delazer, M., 2009b. Flexible transfer of knowledge in 11 mental arithmetic — An fMRI study. Neuroimage 44, 1103-1112.

12 https://doi.org/https://doi.org/10.1016/j.neuroimage.2008.10.025

13 Jacob, S.N., Nieder, A., 2009a. Tuning to non-symbolic proportions in the human frontoparietal 14 cortex. Eur. J. Neurosci. 30, 1432-1442. https://doi.org/10.1111/j.1460-9568.2009.06932.x

15 Jacob, S.N., Nieder, A., 2009b. Notation-Independent Representation of Fractions in the Human

16 Parietal Cortex. J. Neurosci. 29, 4652-4657. https://doi.org/10.1523/JNEUROSCI.065109.2009

18 Jacob, S.N., Vallentin, D., Nieder, A., 2012. Relating magnitudes: The brain's code for proportions.

19 Trends Cogn. Sci. 16, 157-166. https://doi.org/10.1016/j.tics.2012.02.002

20 Jonides, J., Schumacher, E.H., Smith, E.E., Koeppe, R.A., Awh, E., Reuter-Lorenz, P.A.,

21 Marshuetz, C., Willis, C.R., 1998. The role of parietal cortex in verbal working memory. J.

$22 \quad$ Neurosci. 18, 5026-5034.

23 Jordan, K., Heinze, H.J., Lutz, K., Kanowski, M., Jäncke, L., 2001. Cortical activations during the 24 mental rotation of different visual objects. Neuroimage 13, 143-152. 
https://doi.org/10.1006/nimg.2000.0677

2 Klein, E., Moeller, K., Nuerk, H.-C., Willmes, K., 2010. On the neuro-cognitive foundations of basic auditory number processing: An fMRI study. Behav. Brain Funct. 6, 1-13. https://doi.org/10.1186/1744-9081-6-42

5 Knops, A., Thirion, B., Hubbard, E.M., Michel, V., Dehaene, S., 2009. Recruitment of an Area Involved in Eye Movements During Mental Arithmetic. Science (80-. ). 324, 1583-1585.

7 Kriegeskorte, N., Mur, M., Bandettini, P., 2008. Representational similarity analysis - connecting

Mock, J., Huber, S., Bloechle, J., Dietrich, J.F., Bahnmueller, J., Rennig, J., Klein, E., Moeller, K., the branches of systems neuroscience. Front. Syst. Neurosci. 2, 1-28. https://doi.org/10.3389/neuro.06.004.2008

Mussolin, C., Noel, M.P., Pesenti, M., Grandin, C., De Volder, A., 2013. Neural correlates of the numerical distance effect in children. Front. Psychol. 4, 1-9.

Nichols, T., Brett, M., Andersson, J., Wager, T., Poline, J.B., 2005. Valid conjunction inference with the minimum statistic. Neuroimage 25, 653-660. https://doi.org/10.1016/j.neuroimage.2004.12.005

Nieder, A., 2005. Counting on neurons: The neurobiology of numerical competence. Nat. Rev. Neurosci. 6, 177-190. https://doi.org/10.1038/nrn1626

Piazza, M., Mechelli, A., Price, C.J., Butterworth, B., 2006. Exact and approximate judgements of visual and auditory numerosity: An fMRI study. Brain Res. 1106, 177-188. https://doi.org/10.1016/j.brainres.2006.05.104 
and Number Symbols in Human Intraparietal Cortex. Neuron 53, 293-305. https://doi.org/10.1016/j.neuron.2006.11.022

3 Pinel, P., Dehaene, S., Rivière, D., Le Bihan, D., 2001. Modulation of parietal activation by

4 semantic distance in a number comparison task. Neuroimage 14, 1013-1026. https://doi.org/10.1006/nimg.2001.0913

6 Ranganath, C., Johnson, M.K., D’Esposito, M., 2003. Prefrontal activity associated with working 7 memory and episodic long-term memory. Neuropsychologia 41, 378-389. https://doi.org/10.1016/S0028-3932(02)00169-0

9 Ratcliff, R., 1993. Methods for dealing with reaction time outliers. Psychol. Bull. 114, 510-532. https://doi.org/10.1037/0033-2909.114.3.510

11 Ress, D., Backus, B.T., Heeger, D.J., 2000. Activity in primary visual cortex predicts performance

12 in a visual detection task. Nat. Neurosci. 3, 940-945. https://doi.org/10.1038/78856

13 Rushworth, M.F.S., Johansen-Berg, H., Göbel, S.M., Devlin, J.T., 2003. The left parietal and 14 premotor cortices: motor attention and selection. Neuroimage 20, S89-S100. https://doi.org/https://doi.org/10.1016/j.neuroimage.2003.09.011

Rushworth, M.F.S., Krams, M., Passingham, R.E., 2001. The attentional role of the left parietal cortex the district lateralization and localization of motor attention in the human brain. J. Cogn. Neurosci. 13, 698-710.

19 Shuman, M., Kanwisher, N., 2004. Numerical magnitude in the human parietal lobe: Tests of representational generality and domain specificity. Neuron 44, 557-569.

21 Simon, O., Kherif, F., Flandin, G., Poline, J.B., Rivière, D., Mangin, J.-F., Le Bihan, D., Dehaene, 22 S., 2004. Automatized clustering and functional geometry of human parietofrontal networks for 23 language, space, and number. Neuroimage 23, 1192-1202. 
1 Simon, O., Mangin, J.-F., Cohen, L., Le Bihan, D., Dehaene, S., 2002. Topographical Layout of Hand, Eye , Calculation, and Language-Related Areas in the Human Parietal Lobe. Neuron $33,475-487$.

4 Sokolowski, H.M., Fias, W., Mousa, A., Ansari, D., 2017. Common and distinct brain regions in both parietal and frontal cortex support symbolic and nonsymbolic number processing in humans: A functional neuroimaging meta-analysis. Neuroimage 146, 376-394.

Song, J., Jiang, Y., 2006. Visual working memory for simple and complex features : An fMRI study 30, 963-972. https://doi.org/10.1016/j.neuroimage.2005.10.006

9 Sturm, W., Longoni, F., Weis, S., Specht, K., Herzog, H., Vohn, R., Thimm, M., Willmes, K., 2004.

10 Functional reorganisation in patients with right hemisphere stroke after training of alertness: A

11 longitudinal PET and fMRI study in eight cases. Neuropsychologia 42, 434-450.

12 https://doi.org/10.1016/j.neuropsychologia.2003.09.001

13 Thiel, C.M., Zilles, K., Fink, G.R., 2004. Cerebral correlates of alerting, orienting and reorienting of 14 visuospatial attention: An event-related fMRI study. Neuroimage 21, 318-328. https://doi.org/10.1016/j.neuroimage.2003.08.044

16 Uddin, L.Q., Menon, V., 2009. The anterior insula in autism: Under-connected and under-examined. Neurosci. Biobehav. Rev. 33, 1198-1203. https://doi.org/https://doi.org/10.1016/j.neubiorev.2009.06.002

19 Venkatraman, V., Ansari, D., Chee, M.W.L., 2005. Neural correlates of symbolic and non-symbolic $20 \quad$ arithmetic. Neuropsychologia 43, 744-753.

21 Wojciulik, E., Kanwisher, N., 1999. The Generality of Parietal Involvementin Visual Attention. $22 \quad$ Neuron 23, 1-18. 


\section{$1 \quad$ Figure legend}

3 Figure 1: A Speed and B accuracy in the four conditions (dot patterns, fractions, pie charts, and 4 decimals).

6 Figure 2. Significant joint activation for proportion processing across the four conditions (e.g.,

7 fractions, decimals, dot patterns, pie charts) is given in red. The blue clusters indicate significant 8 activation specific to overall magnitude processing as reflected by the numerical distance effect across

9 the four presentation formats in a nearby, but different brain region (Mock et al., 2018). Activations 10 were thresholded at a whole-brain FWE-corrected $p$-value of $<.05$.

12 Figure 3. Contrast between part-whole proportions (i.e., fractions, dot patterns, and pie charts) and 13 decimals (part-whole - decimals). Significant activation depicts part-whole relation processing 14 without base-10 related processing. Activations were thresholded at a whole-brain FWE-corrected $p$ 15 value of $<.05$.

16

17 Figure 4. Percent signal change of the four presentation formats of the clusters in the inferior parietal 18 cortex for left and right hemisphere. Error bars indicate $+/-1$ standard error of the mean.

19

20 Figure 5. Min-max normalized Representational Similarity Matrix for the four different notation 21 formats with 1 being most similar. 
1 Figure 6. Dendrograms for left and right hemisphere of the agglomerative hierarchical cluster analysis

2 on the mean z-standardized $\beta$ estimates for the four different notation formations. Distance indicates

3 the Euclidean distance with lower distance being more similar.

4

5 Figure 7. Experimental procedure at the beginning of each block (i.e., one out of five trials).

6

7 Figure 8. Example stimuli (4/7 vs. 2/3) for the four different presentation formats. A depicts decimals,

8 B fractions, $\mathrm{C}$ dot patterns, and D pie charts.

9

10

11

12

13 
Figures

2

$3 \quad$ Figure 1

4
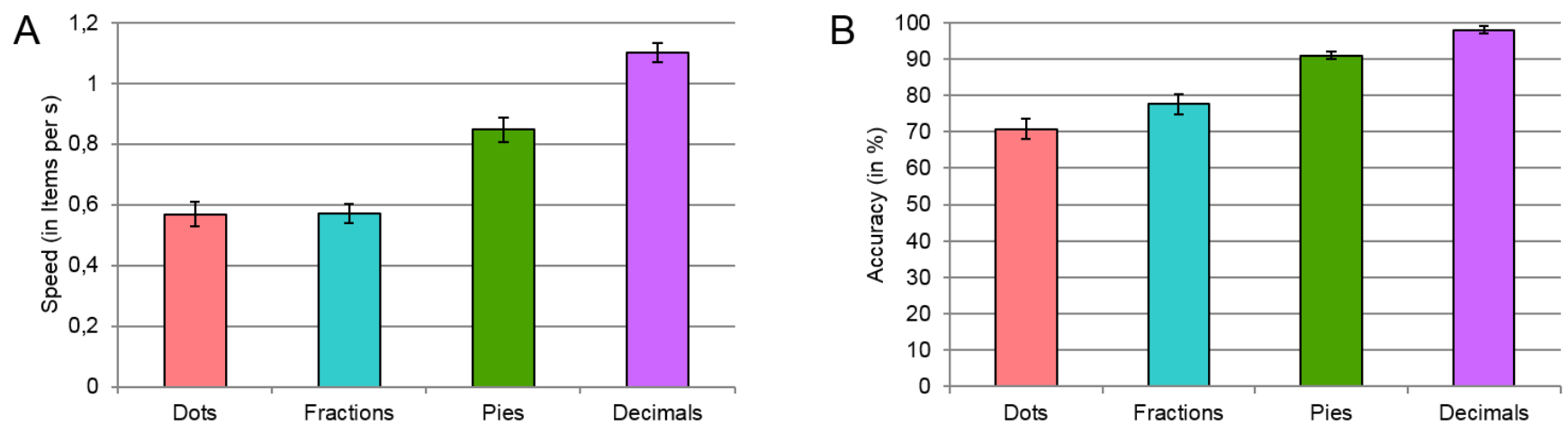

5

$6 \quad$ Figure 2

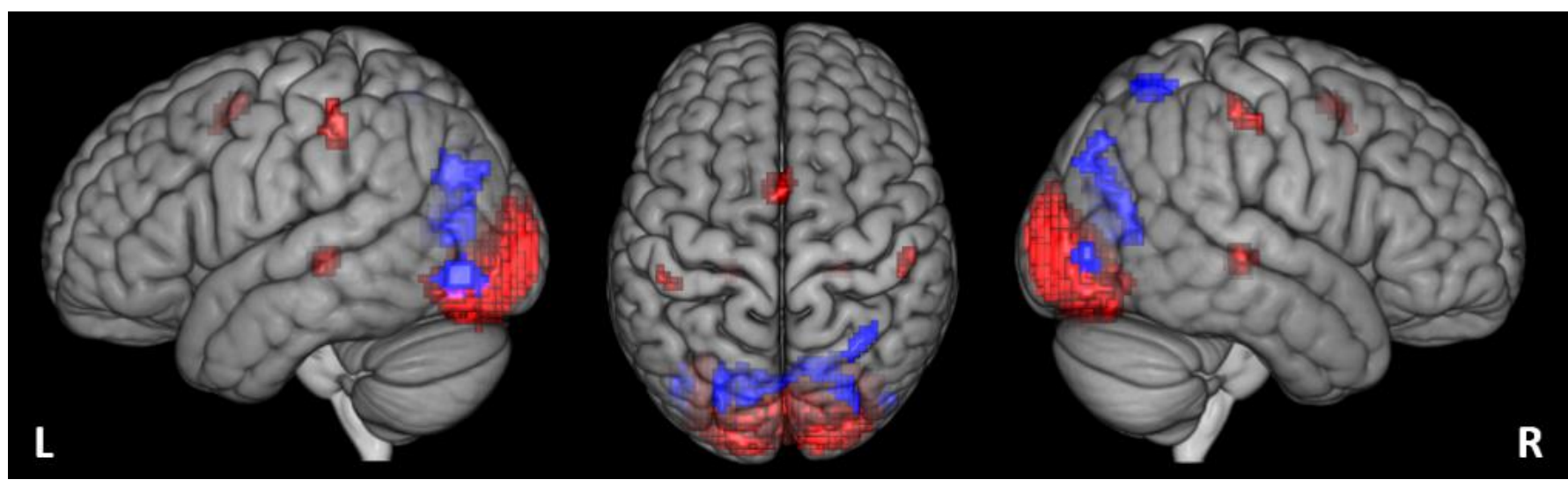

8 
$1 \quad$ Figure 3

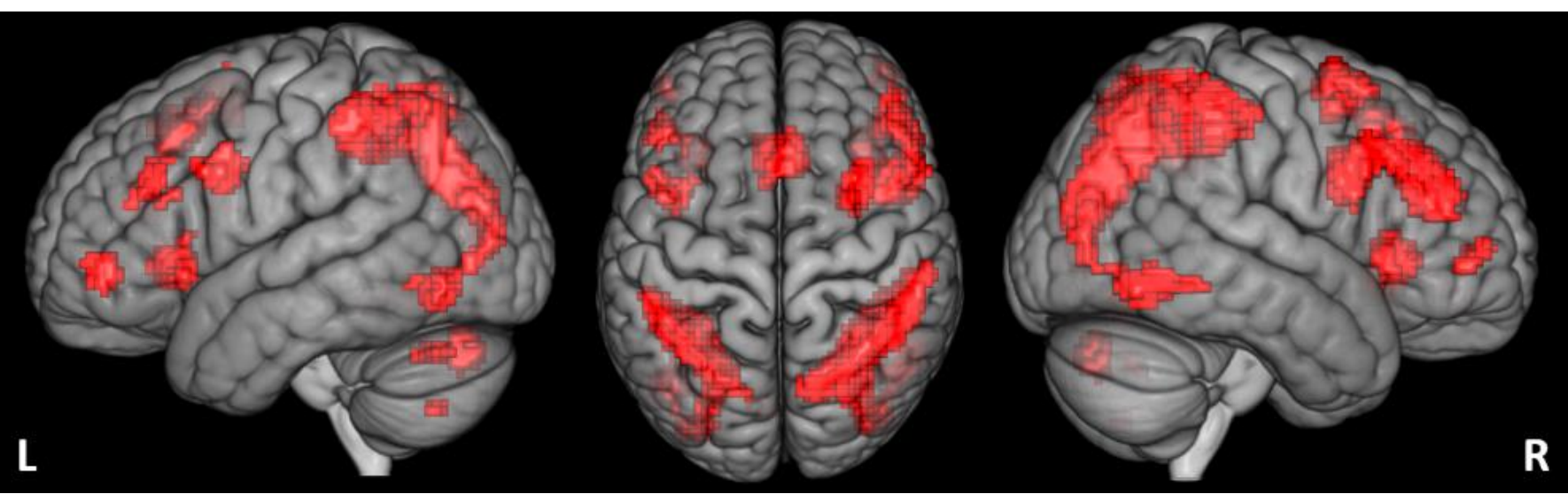

3

4

$5 \quad$ Figure 4

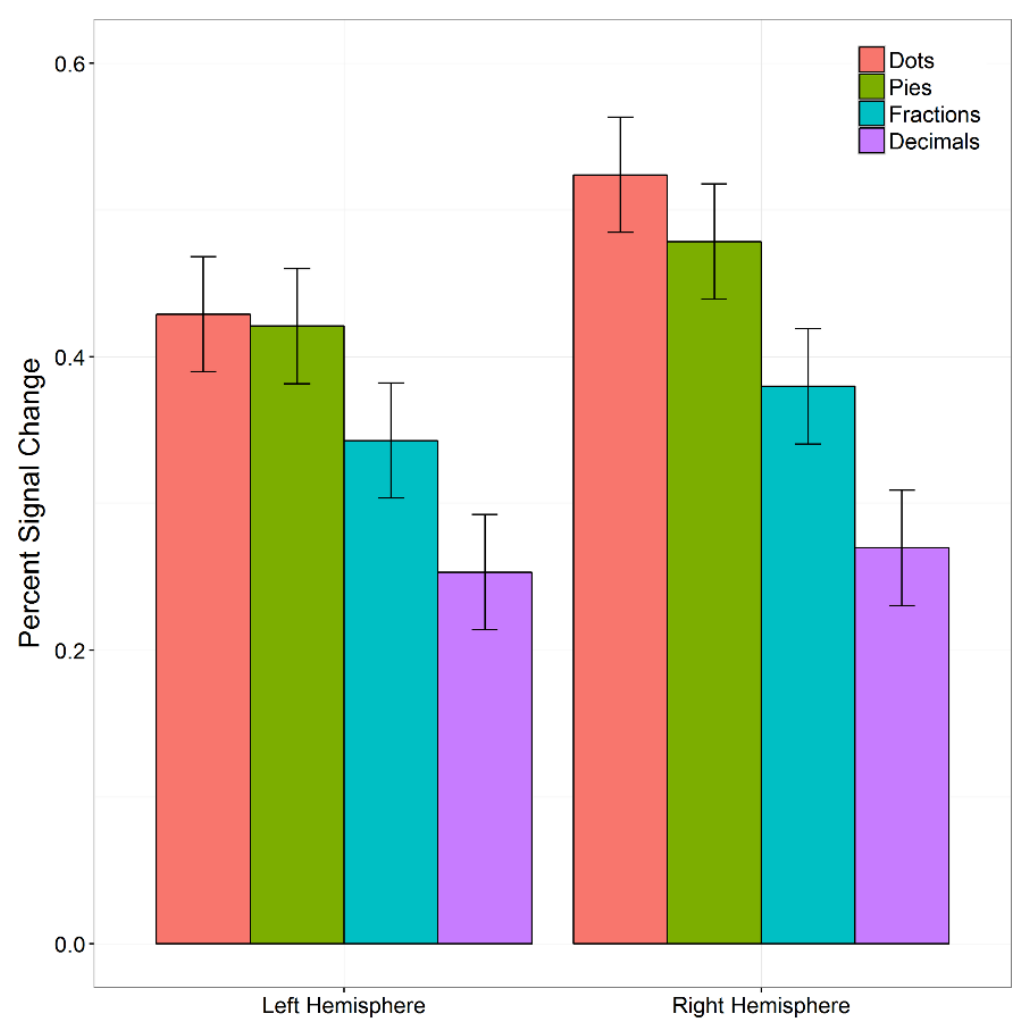

7 


\section{$1 \quad$ Figure 5}

\section{Left Hemisphere}

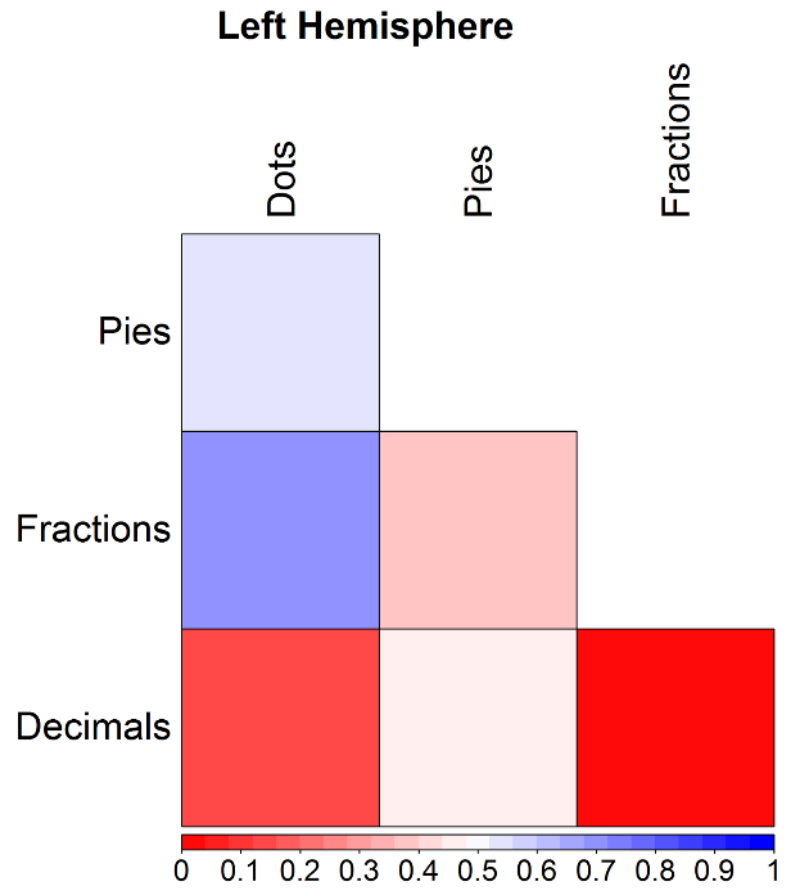

\section{Right Hemisphere}

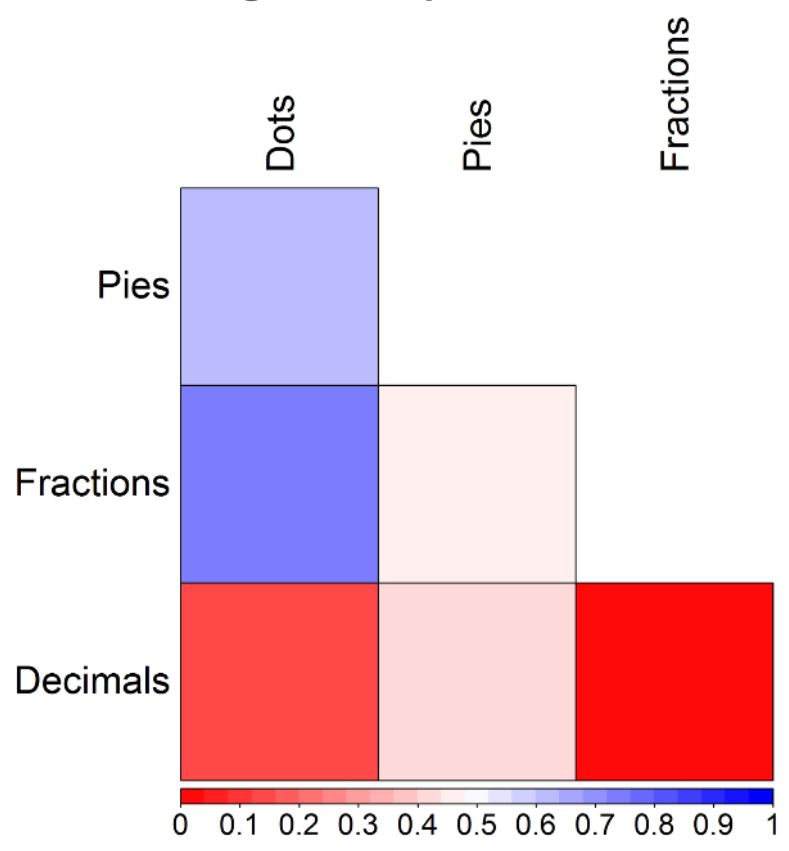

3

4

$5 \quad$ Figure 6

6

Left Hemisphere

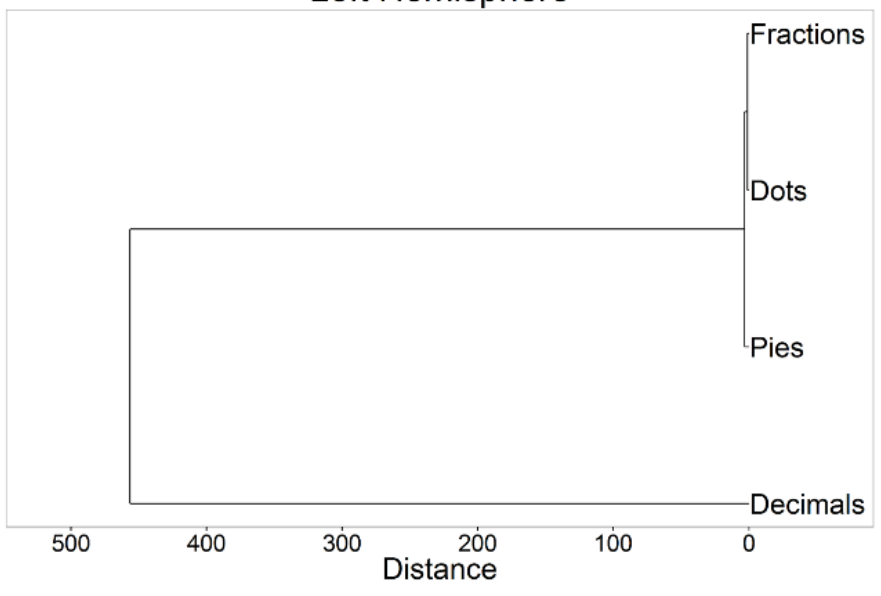

Right Hemisphere

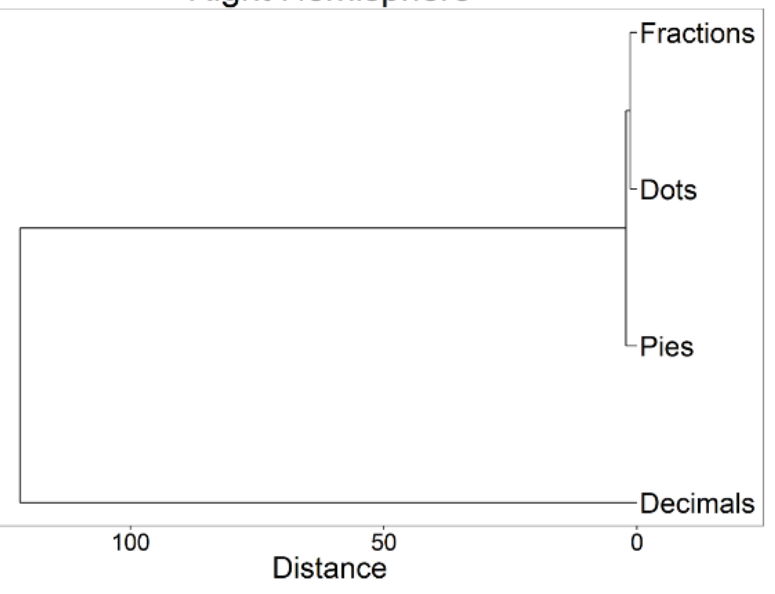


$\begin{array}{ll}2 & \text { Figure } 7\end{array}$

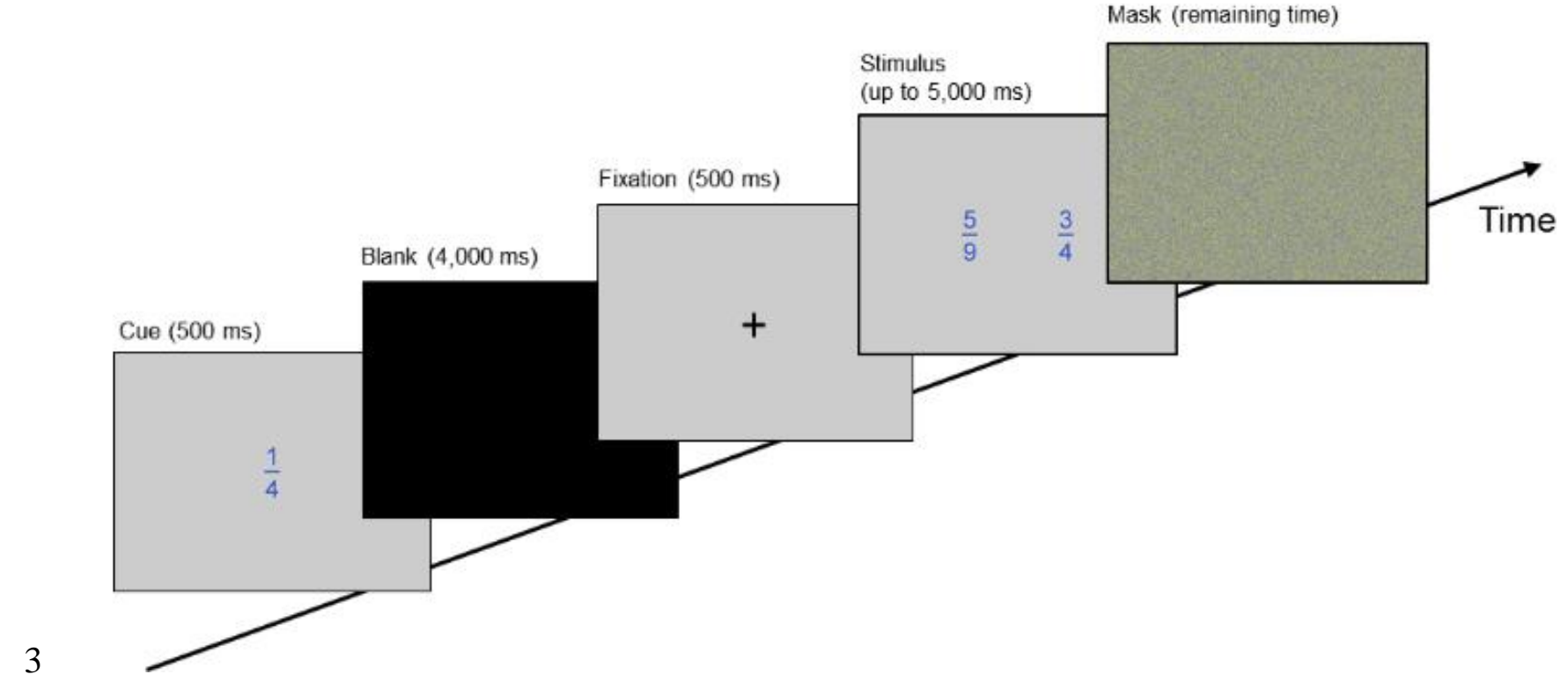

4

5

6

$7 \quad$ Figure 8

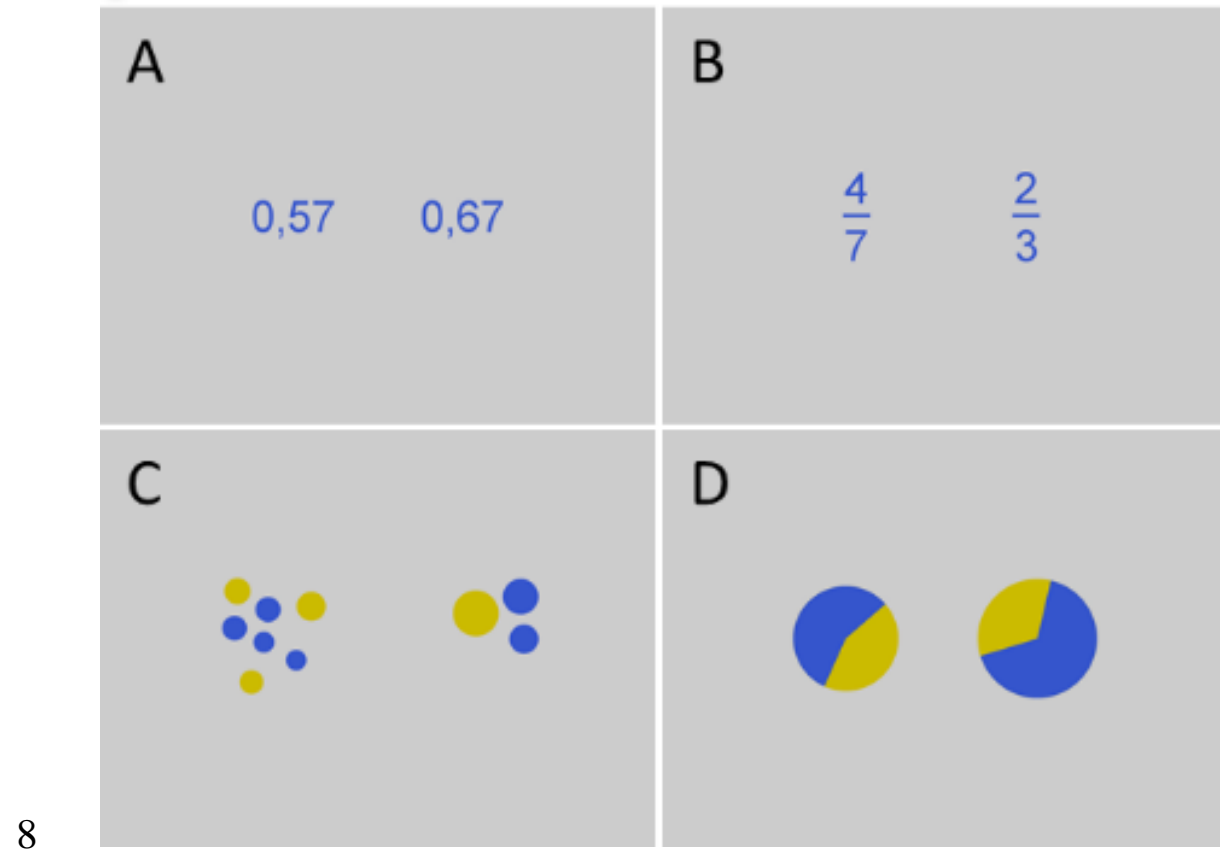


Table

2 Table 1. Joint activations across the four conditions (i.e., fractions, decimals, dot patterns, pie charts)

3 as revealed by the conjunction analysis. Activations were thresholded at a whole-brain FWE $p$-value

4 of $<.05$ with a cluster size of $k=10$ voxels.

\begin{tabular}{|c|c|c|c|c|c|c|}
\hline \multirow{2}{*}{$\begin{array}{l}\text { Contrast } \\
\text { Conjunction }\end{array}$} & \multirow{2}{*}{$\begin{array}{l}\text { Brain region } \\
\text { LH inferior parietal lobule }\end{array}$} & \multicolumn{3}{|c|}{ MNI (x, y, z) } & \multirow{2}{*}{$\begin{array}{l}k \\
40\end{array}$} & \multirow{2}{*}{$\begin{array}{c}t \\
6.08\end{array}$} \\
\hline & & -45 & -32 & 43 & & \\
\hline & RH inferior parietal lobule & 51 & -25 & 48 & 28 & 5.74 \\
\hline & LH thalamus & -22 & -27 & -3 & 39 & 11.73 \\
\hline & RH thalamus & 23 & -27 & -3 & 42 & 10.21 \\
\hline & RH supplementary area & 1 & 6 & 53 & 56 & 7.86 \\
\hline & RH lingual gyrus & 16 & -97 & -8 & 3637 & 25.15 \\
\hline & LH lingual gyrus* & -10 & -90 & 15 & & 17.67 \\
\hline & RH calcarine gyrus* & 13 & -90 & 3 & & 17.24 \\
\hline & LH calcarine gyrus* & -5 & -90 & -5 & & 18.17 \\
\hline & LH middle occipital gyrus* & -22 & 100 & 3 & & 20.37 \\
\hline & RH middle occipital gyrus ${ }^{*}$ & 16 & 102 & 8 & & 19.57 \\
\hline & LH inferior occipital gyrus* & -22 & -95 & 10 & & 22.95 \\
\hline & RH inferior occipital gyrus & 31 & -85 & 10 & & 16.11 \\
\hline
\end{tabular}

$6 \mathrm{RH}=$ right hemisphere; FWE-corrected $p$-value $<.05$, cluster size $k=10$ voxels; $t=t$-value. ${ }^{*}$ Minor 7 maximum.

8

9 Table 2. Activations for part-whole processing as revealed by the contrast part-whole (i.e., fractions,

10 pie charts, and dot patterns) vs. decimals. Activations were thresholded at a whole-brain FWE $p$-value 11 of $<.05$ with a cluster size of $k=10$ voxels. 
$\begin{array}{lll}\text { Contrast } & \text { BNI (x, y, z) } \quad k \quad t\end{array}$

\begin{tabular}{|c|c|c|c|c|c|c|}
\hline Part-whole & RH Inferior parietal lobule (hIP2) & 46 & -40 & 50 & 1825 & 10.99 \\
\hline \multirow[t]{18}{*}{ vs. decimals } & RH Inferior parietal lobule (IPL)* & 51 & -37 & 53 & & 10.59 \\
\hline & RH Superior occipital gyrus* & 28 & -65 & 40 & & 9.53 \\
\hline & RH Superior parietal lobule* & 26 & -67 & 40 & & 9.34 \\
\hline & LH Inferior parietal lobule (hIP3) & -35 & -50 & 48 & 1164 & 8.48 \\
\hline & LH Inferior parietal lobule (IPL)* & -47 & -37 & 48 & & 8.36 \\
\hline & LH Middle occipital gyrus* & -25 & -65 & 38 & & 8.34 \\
\hline & LH Inferior parietal lobule (hIP1)* & -32 & -47 & 43 & & 8.30 \\
\hline & RH Middle frontal gyrus & 43 & 36 & 20 & 633 & 10.70 \\
\hline & RH Inferior frontal gyrus* & 48 & 13 & 30 & & 8.96 \\
\hline & RH Supplementary motor area & 6 & 26 & 45 & 267 & 8.96 \\
\hline & LH Supplementary motor area* & -7 & 21 & 45 & & 8.11 \\
\hline & RH Insula & 33 & 28 & -3 & 169 & 10.46 \\
\hline & RH Middle frontal gyrus & 31 & 11 & 60 & 166 & 7.35 \\
\hline & LH Precentral gyrus (Area 44) & -42 & 3 & 33 & 153 & 6.71 \\
\hline & LH Insula & -32 & 21 & -3 & 121 & 8.49 \\
\hline & LH Inferior frontal gyrus (Area 45) & -47 & 28 & 30 & 88 & 6.55 \\
\hline & LH Middle orbital gyrus & -42 & 46 & -3 & 68 & 6.45 \\
\hline & RH Middle orbital gyrus & 38 & 53 & -3 & 46 & 6.34 \\
\hline
\end{tabular}

1 Abbreviations: $k=$ cluster size; LH = left hemisphere; MNI: Montreal Neurological Institute; RH =

2 right hemisphere; FWE-corrected $p$-value $<.05$, cluster size $k=10$ voxels; $t=\mathrm{t}$-value. $*$ Minor 3 maximum. 


\section{Appendix}

2

3 Table A1. Activations found for magnitude comparisons with fractions. Activations were thresholded

4 at a whole-brain FWE $p$-value of $<.05$ with a cluster size of $k=10$ voxels.

\begin{tabular}{|c|c|c|c|c|c|c|}
\hline \multirow{2}{*}{$\begin{array}{l}\text { Contrast } \\
\text { Fractions }\end{array}$} & \multirow{2}{*}{$\begin{array}{l}\text { Brain region } \\
\text { LH Inferior parietal lobule }\end{array}$} & \multicolumn{3}{|c|}{$\mathrm{MNI}(\mathrm{x}, \mathrm{y}, \mathrm{z})$} & \multirow{2}{*}{$\begin{array}{l}k \\
1101\end{array}$} & \multirow{2}{*}{$\begin{array}{l}t \\
12.07\end{array}$} \\
\hline & & -47 & -37 & 48 & & \\
\hline VS. & LH Intraparietal sulcus (hIP3)* & -32 & -50 & 48 & & 11.23 \\
\hline \multirow[t]{16}{*}{ Baseline } & LH Superior parietal lobule* & -25 & -62 & 45 & & 10.62 \\
\hline & RH Intraparietal sulcus (hIP2) & 46 & -40 & 48 & 1052 & 10.82 \\
\hline & RH Intraparietal sulcus (hIP3) ${ }^{*}$ & 36 & -50 & 48 & & 10.48 \\
\hline & RH Intraparietal sulcus (hIP1) ${ }^{*}$ & 28 & -55 & 48 & & 9.15 \\
\hline & LH Precentral Gyrus (Area 44) & -45 & 6 & 30 & 679 & 11.04 \\
\hline & LH Inferior frontal gyrus (Area 45) ${ }^{*}$ & -45 & 31 & 23 & & 7.42 \\
\hline & RH Inferior frontal gyrus (Area 44) & 46 & 6 & 25 & 311 & 10.39 \\
\hline & RH Middle frontal gyrus (Area 45) & 48 & 36 & 23 & 225 & 9.94 \\
\hline & RH Superior frontal gyrus & 28 & 1 & 53 & 149 & 8.30 \\
\hline & LH Middle frontal gyrus & -25 & 1 & 55 & 90 & 7.65 \\
\hline & LH Inferior frontal gyrus & -47 & 43 & 10 & 53 & 5.90 \\
\hline & RH Insula & 33 & 26 & 0 & 170 & 10.64 \\
\hline & LH Insula & -30 & 23 & 3 & 127 & 9.56 \\
\hline & LH Thalamus & -22 & -27 & -3 & 60 & 13.12 \\
\hline & RH Thalamus & 23 & -27 & -3 & 51 & 10.55 \\
\hline & RH supplementary area & 6 & 18 & 48 & 431 & 10.72 \\
\hline
\end{tabular}




\begin{tabular}{|c|c|c|c|c|c|}
\hline RH Lingual gyrus & 16 & -97 & -8 & 4891 & 25.15 \\
\hline RH Calcarine gyrus ${ }^{*}$ & 23 & -100 & -3 & & 24.89 \\
\hline LH Inferior occipital gyrus* & -22 & -95 & -10 & & 22.95 \\
\hline LH Inferior occipital gyrus* & -12 & -100 & -8 & & 22.21 \\
\hline LH Middle occipital gyrus* & -15 & -92 & -8 & & 21.44 \\
\hline LH Middle occipital gyrus* & -22 & -100 & 3 & & 20.37 \\
\hline RH Cuneus* & 16 & -102 & 8 & & 19.57 \\
\hline LH Calcarine gyrus* & -5 & -90 & -5 & & 18.17 \\
\hline LH Lingual gyrus* & -10 & -90 & -15 & & 17.67 \\
\hline RH Calcarine gyrus* & 13 & -90 & 3 & & 17.24 \\
\hline LH Cerebelum (VII) & -32 & -67 & -50 & 26 & 7.23 \\
\hline
\end{tabular}

1 Abbreviations: $\mathrm{k}=$ cluster size; $\mathrm{LH}=$ left hemisphere; MNI: Montreal Neurological Institute; $\mathrm{RH}=$ 2 right hemisphere; $\mathrm{t}=\mathrm{t}$-value. $*$ Minor maximum.

3

4 Table A2. Activations found for magnitude comparison with decimals. Activations were thresholded 5 at a whole-brain FWE $p$-value of $<.05$ with a cluster size of $k=10$ voxels.

\begin{tabular}{|c|c|c|c|c|c|c|}
\hline \multirow{2}{*}{$\begin{array}{l}\text { Contrast } \\
\text { Decimals }\end{array}$} & \multirow{2}{*}{$\begin{array}{l}\text { Brain region } \\
\text { LH Inferior parietal lobule }\end{array}$} & \multicolumn{3}{|c|}{$\mathrm{MNI}(\mathrm{x}, \mathrm{y}, \mathrm{z})$} & \multirow{2}{*}{$\begin{array}{l}k \\
66\end{array}$} & \multirow{2}{*}{$\begin{array}{l}t \\
6.08\end{array}$} \\
\hline & & -45 & -32 & 43 & & \\
\hline VS. & LH Postcentral Gyrus* & -50 & -27 & 55 & & 5.73 \\
\hline \multirow[t]{3}{*}{ Baseline } & RH Postcentral Gyrus & 51 & -25 & 48 & 40 & 5.74 \\
\hline & LH Thalamus & -22 & -17 & -3 & 58 & 11.73 \\
\hline & RH supplementary motor area & 1 & 6 & 53 & 169 & 7.86 \\
\hline
\end{tabular}




$\begin{array}{lccccc}\text { RH Calcarine gyrus } & 16 & -97 & -5 & 3797 & 30.74 \\ \text { LH Middle occipital gyrus }^{*} & -15 & -92 & -8 & 28.40 \\ \text { LH Inferior Occipital gyrus }^{*} & -12 & -100 & -8 & 27.65 \\ \text { LH Calcarine gyrus }^{*} & -17 & -100 & -5 & 26.87 \\ \text { RH Cuneus* }^{*} & 16 & -102 & 8 & 22.10\end{array}$

1 Abbreviations: $k=$ cluster size; LH = left hemisphere; MNI: Montreal Neurological Institute;

$2 \mathrm{RH}=$ right hemisphere; $t=t$-value. ${ }^{*}$ Minor maximum.

4 Table A3. Activations found for magnitude comparison with dot patterns. Activations were 5 thresholded at a whole-brain FWE $p$-value of $<.05$ with a cluster size of $k=10$ voxels.

\begin{tabular}{|c|c|c|c|c|c|c|}
\hline \multirow{2}{*}{$\begin{array}{l}\text { Contrast } \\
\text { Dot patterns }\end{array}$} & \multirow{2}{*}{$\begin{array}{l}\text { Brain region } \\
\text { RH Calcarine gyrus }\end{array}$} & \multicolumn{3}{|c|}{ MNI (x, y, z) } & \multirow{2}{*}{$\begin{array}{l}k \\
9332\end{array}$} & \multirow{2}{*}{$\begin{array}{l}t \\
28.43\end{array}$} \\
\hline & & 16 & -97 & -5 & & \\
\hline vs. & RH Intraparietal sulcus (hIP2) ${ }^{* *}$ & 46 & -35 & 48 & 716 & 12.84 \\
\hline \multirow[t]{9}{*}{ Baseline } & RH Intraparietal sulcus (hIP3) ${ }^{* *}$ & 36 & -50 & 50 & & 12.59 \\
\hline & LH Inferior parietal lobule ${ }^{* *}$ & -47 & -37 & 48 & 480 & 13.24 \\
\hline & LH Intraparietal sulcus (hIP3) ${ }^{* *}$ & -32 & -50 & 48 & & 11.53 \\
\hline & LH Middle occipital gyrus* & -15 & -92 & -8 & & 26.54 \\
\hline & RH Lingual gyrus* & 18 & -90 & -8 & & 26.16 \\
\hline & RH Superior occipital gyrus ${ }^{*}$ & 23 & -97 & 5 & & 25.69 \\
\hline & LH Inferior occipital gyrus* & -12 & -100 & -8 & & 25.26 \\
\hline & LH Calcarine gyrus* & -17 & -100 & -5 & & 24.73 \\
\hline & RH Cuneus* & 16 & -100 & 10 & & 21.79 \\
\hline
\end{tabular}




\begin{tabular}{|c|c|c|c|c|c|}
\hline RH Precentral gyrus & 43 & 6 & 28 & 772 & 11.25 \\
\hline RH Middle frontal gyrus* & 43 & 36 & 18 & & 10.82 \\
\hline RH Inferior frontal gyrus (Area 45) ${ }^{*}$ & 48 & 13 & 30 & & 10.44 \\
\hline LH Precentral gyrus & -42 & 1 & 33 & 359 & 9.36 \\
\hline RH Superior frontal gyrus & 28 & 1 & 53 & 326 & 9.33 \\
\hline LH Superior frontal gyrus & -25 & -2 & 53 & 152 & 8.45 \\
\hline LH Inferior frontal gyrus & -37 & 28 & 20 & 143 & 6.91 \\
\hline LH Middle frontal gyrus & -45 & 53 & 0 & 110 & 6.18 \\
\hline RH Middle frontal gyrus & 38 & 58 & 8 & 100 & 5.31 \\
\hline RH Insula & 33 & 26 & 0 & 251 & 11.03 \\
\hline RH Putamen* & 23 & 16 & 0 & & 5.23 \\
\hline LH Insula & -30 & 23 & 3 & 187 & 9.81 \\
\hline LH Putamen & -22 & 6 & 3 & 58 & 5.60 \\
\hline RH Thalamus & 23 & -27 & -3 & 54 & 10.22 \\
\hline LH Thalamus & -22 & -27 & -3 & 47 & 11.83 \\
\hline RH supplementary area & 6 & 18 & 48 & 543 & 11.59 \\
\hline LH Cerebellum (VIII) & -30 & -67 & -50 & 73 & 8.79 \\
\hline RH Cerebellar Vermis (9) & 1 & -55 & -35 & 27 & 7.44 \\
\hline
\end{tabular}

$2 \mathrm{RH}=$ right hemisphere; $t=t$-value. ${ }^{*}$ Minor maximum; ${ }^{* *}$ Secondary peak.

4 Table A4. Activations found for magnitude comparison with pie charts. Activations were thresholded 5 at a whole-brain FWE $p$-value of $<.05$ with a cluster size of $k=10$ voxels. 
$\begin{array}{lll}\text { Contrast } & \text { BNI (x, y, z) } \quad k \quad t\end{array}$

\begin{tabular}{|c|c|c|c|c|c|c|}
\hline Pie charts & RH Postcentral gyrus & 48 & -25 & 45 & 989 & 10.10 \\
\hline vs. & RH Superior parietal lobule (hIP3)* & 33 & -45 & 48 & & 8.07 \\
\hline \multirow[t]{18}{*}{ Baseline } & $\mathrm{RH}$ Inferior parietal lobule (hIP2) ${ }^{*}$ & 41 & -40 & 50 & & 7.85 \\
\hline & RH Inferior parietal lobule (hIP3) ${ }^{*}$ & 31 & -55 & 53 & & 7.35 \\
\hline & LH Postcentral gyrus & -42 & -35 & 43 & 665 & 9.40 \\
\hline & LH Superior parietal lobule (hIP3) ${ }^{*}$ & -37 & -45 & 58 & & 7.77 \\
\hline & LH Inferior parietal lobule (hIP3) * & -32 & -45 & 48 & & 7.06 \\
\hline & RH Middle frontal gyrus & 31 & -2 & 55 & 103 & 6.90 \\
\hline & RH Superior frontal gyrus ${ }^{*}$ & 28 & -5 & 63 & & 6.31 \\
\hline & RH Inferior frontal gyrus & 46 & 6 & 25 & 95 & 6.85 \\
\hline & LH Precentral gyrus & -50 & 3 & 30 & 66 & 6.42 \\
\hline & LH Thalamus & -22 & -27 & -3 & 74 & 13.64 \\
\hline & RH Thalamus & 23 & -27 & -3 & 59 & 10.54 \\
\hline & LH Putamen & -22 & 8 & 3 & 54 & 6.01 \\
\hline & RH supplementary area & 1 & 6 & 53 & 258 & 8.76 \\
\hline & RH Calcarine gyrus & 16 & -97 & -5 & 5439 & 28.29 \\
\hline & LH Calcarine gyrus* & -12 & -92 & -8 & & 26.54 \\
\hline & LH Inferior occipital gyrus* & -12 & -100 & -8 & & 25.75 \\
\hline & RH Superior occipital gyrus* & 23 & -97 & 5 & & 24.94 \\
\hline & LH Calcarine gyrus* & -17 & -100 & -5 & & 24.81 \\
\hline
\end{tabular}

1 Abbreviations: $k=$ cluster size; $\mathrm{LH}=$ left hemisphere; MNI: Montreal Neurological Institute; $\mathrm{RH}=$ 2 right hemisphere; $t=\mathrm{t}$-value. ${ }^{*}$ Minor maximum. 